\title{
MODIFIED THREE-POINT DIFFERENCE SCHEMES OF HIGH-ACCURACY ORDER FOR SECOND ORDER NONLINEAR ORDINARY DIFFERENTIAL EQUATIONS
}

\author{
MYROSLAV V. KUTNIV \\ Lviv Polytechnical National University \\ 12 St. Bandery Str., 79013 Lviv, Ukraine
}

\begin{abstract}
This work discusses issues on the design and analysis of three-point difference schemes for the nonlinear boundary-value problem. A new effective implementation of an exact three-point difference schemes on irregular grids in terms of homogeneous three-point difference schemes of a high-accuracy order is proposed.
\end{abstract}

2000 Mathematics Subject Classification: 65L10; 65L12; 65L20.

Keywords: nonlinear boundary-value problem, three-point difference scheme, linearization method and the principle of contraction mapping.

\section{Introduction}

In [6], for a nonlinear boundary-value problem of the form

$$
\frac{d}{d x}\left[k(x) \frac{d u}{d x}\right]=-f(x, u(x)), \quad x \in(0,1), \quad u(0)=\mu_{1}, \quad u(1)=\mu_{2}
$$

with piecewise smooth $k(x), f(x, u)$ for the first an approach to the construction of exact three-point difference schemes (ETDS) was proposed, and ways of their implementation in terms of $m$ th order accurate three-point difference schemes (TDS) were shown . Further development of the ideas of [6] was obtained in [5] and in the case of nonlinear monotone ODE in [2]. High-accuracy order TDS for systems of nonlinear ODE are constructed in [3,4].

In this work, we propose a new effective implementation of an ETDS on an irregular grid $\hat{\omega}_{h}$ in terms of a homogeneous TDS of the order of accuracy $\bar{m}=2[(m+1) / 2]$ ([·] is the integer part) that approximate the function $u(x)$ and its flux $k(x) \frac{d u(x)}{d x}$. The existence and uniqueness of the solution of the ETDS and truncated TDS of rank $\bar{m}$ is proved, the estimate of the accuracy of the TDS is given under weaker conditions than in [5]. To construct an $\bar{m}$ th order accurate TDS, like the $m$ th order accurate TDS in $[5,6]$, at each point $x_{j}, j=1,2, \ldots, N-1$ of the grid $\hat{\omega}_{h}$ it is necessary to solve two nonlinear initial value problems on the intervals $\left[x_{j-1}, x_{j}\right]$ (forward) and $\left[x_{j}, x_{j+1}\right]$ (backward). Each initial value problem is solved in one step by the $\bar{m}$ th order accurate one-step method (Taylor series or the Runge-Kutta method). The efficiency of the fourth-order and six-order accurate TDS is illustrated by a numerical example. This work modifies and develops the results of $[5,6]$. 


\section{Existence and uniqueness of the solution to the problem}

In this paper, the linearization method and the principle of contraction mapping are taken as the basis (see, e.g., [1]). With the help of this method we prove the existence and uniqueness theorem for the solution to problem (1.1), which is used below to prove existence of ETDS and analyse truncated TDS. This theorem is not the strongest among known in the literature, however, it allows us to achieve the main purpose of this paper connected with the further development of the theory TDS of arbitrary order of accuracy.

Theorem 2.1. Let conditions

$$
\begin{aligned}
& 0<c_{1} \leqslant k(x) \quad \forall x \in[0,1], \quad k(x) \in Q^{1}[0,1], \\
& f_{u}(x) \equiv f(x, u) \in Q^{0}[0,1], \quad|f(x, u)| \leqslant K \quad \forall x \in[0,1], \quad u \in \Omega([0,1], r), \\
& |f(x, u)-f(x, v)| \leqslant L|u-v| \quad \forall x \in[0,1], \quad u, v \in \Omega([0,1], r), \\
& q=L / c_{1}<1
\end{aligned}
$$

be fulfilled. Then, problem (1.1) has a unique solution $u(x)$ in $\Omega([0,1], r)$, which can be found by the method of successive approximations

$$
\begin{gathered}
\frac{d}{d x}\left[k(x) \frac{d u^{(n)}}{d x}\right]=-f\left(x, u^{(n-1)}(x)\right), x \in(0,1) \\
u^{(n)}(0)=\mu_{1}, u^{(n)}(1)=\mu_{2}, n=1,2, \ldots, u^{(0)}(x)=\frac{V_{2}(x)}{V_{1}(1)} \mu_{1}+\frac{V_{1}(x)}{V_{1}(1)} \mu_{2}
\end{gathered}
$$

with the error estimate

$$
\left\|u^{(n)}-u\right\|_{1, \infty,(0,1)} \leqslant \frac{q^{n}}{1-q} r .
$$

Here $Q^{p}[0,1]$ is the class of functions with piecewise continuous derivatives up to the $p$ th order, including the finite number of discontinuity points of the first kind; $\Omega([0,1], r)$ is a set of functions of the form

$$
\begin{aligned}
\Omega([0,1], r) & =\left\{u(x): u(x) \in W_{\infty}^{1}(0,1), \quad u(x), k(x) \frac{d u}{d x} \in C[0,1], \quad\left\|u-u^{(0)}\right\|_{1, \infty,(0,1)} \leqslant r\right\}, \\
\|u\|_{0, \infty,(0,1)} & =\underset{x \in(0,1)}{\operatorname{vraimax}}|u(x)|,\|u\|_{1, \infty,(0,1)}=\max \left\{\|u\|_{0, \infty,(0,1)},\left\|\frac{d u}{d x}\right\|_{0, \infty,(0,1)}\right\}, \\
r & =\frac{K}{c_{1}}, \quad V_{1}(x)=\int_{0}^{x} \frac{d t}{k(t)}, V_{2}(x)=\int_{x}^{1} \frac{d t}{k(t)} .
\end{aligned}
$$

Proof. We write problem (1.1) in an equivalent integral form

$$
u(x)=\Re(x, u(\cdot))=\int_{0}^{1} G(x, \xi) f(\xi, u(\xi)) d \xi+u^{(0)}(x), \quad 0 \leqslant x \leqslant 1,
$$

where

$$
G(x, \xi)=\frac{1}{V_{1}(1)} \begin{cases}V_{1}(x) V_{2}(\xi), & 0 \leqslant x \leqslant \xi \\ V_{2}(x) V_{1}(\xi), & \xi \leqslant x \leqslant 1\end{cases}
$$


Since $V_{1}(x)>0$ monotonically increases and $V_{2}(x)>0$ monotonically decreases, $V_{\alpha}(x) \leqslant$ $V_{1}(1) \leqslant 1 / c_{1}, x \in[0,1], \alpha=1,2$. Therefore, for the Green function $G(x, \xi)$, the following estimates are valid:

$$
G(x, \xi) \leqslant \frac{1}{c_{1}}, \quad\left|\frac{\partial G(x, \xi)}{\partial x}\right| \leqslant \frac{1}{c_{1}} .
$$

We show that operator $(2.7)$ maps the set $\Omega([0,1], r)$ onto itself. Taking into account relations (2.9) and condition (2.2), we obtain

$$
\left\|\Re(x, v(\cdot))-u^{(0)}\right\|_{1, \infty,(0,1)} \leqslant \frac{1}{c_{1}} \int_{0}^{1}|f(\xi, v)| d \xi \leqslant \frac{K}{c_{1}}, \quad \forall v \in \Omega([0,1], r) .
$$

Moreover, $\Re(x, u(\cdot))$ is a contraction operator on $\Omega([0,1], r)$ because

$$
\begin{aligned}
\|\Re(x, u(\cdot))-\Re(x, v(\cdot))\|_{1, \infty,(0,1)} & \leqslant \frac{1}{c_{1}} \int_{0}^{1}|f(\xi, u(\xi))-f(\xi, v(\xi))| d \xi \\
& \leqslant q\|u-v\|_{1, \infty,(0,1)}, \quad \forall u, v \in \Omega([0,1], r) .
\end{aligned}
$$

Thus, for the operator $\Re(x, u(\cdot))$, for $q=L / c_{1}<1$, all conditions of the principle of contraction mapping are fulfilled and, hence, problem (1.1) has a unique solution, which can be obtained by the method of successive approximations (2.5) with the error estimate (2.6). The way of obtaining of the estimate (2.6) is standard (see, e.g., [9]), and, therefore, we do not describe it.

\section{Existence of an exact three-point difference scheme}

On the closed interval $[0,1]$ we introduce an irregular grid

$$
\hat{\bar{\omega}}_{h}=\left\{x_{j} \in[0,1], j=0,1, \ldots, N, x_{0}=0, x_{N}=1, h_{j}=x_{j}-x_{j-1}>0, \sum_{j=1}^{N} h_{j}=1\right\}
$$

such that the discontinuity points of functions $k(x), f(x, u)$ coincide with the nodes of the grid $\hat{\omega}_{h}=\left\{x_{j}, j=1,2, \ldots, N-1\right\}$. Denote by $\rho$ the set of all discontinuity points and assume that $N$ is such that $\rho \subseteq \hat{\omega}_{h}$. At the discontinuity points the usual consistency conditions

$$
u\left(x_{i}-0\right)=u\left(x_{i}+0\right),\left.\quad k(x) \frac{d u}{d x}\right|_{x=x_{i}-0}=\left.k(x) \frac{d u}{d x}\right|_{x=x_{i}+0} \forall x_{i} \in \rho
$$

must be satisfied

Let us introduce the set

$$
\Omega\left(\hat{\bar{\omega}}_{h}, r\right)=\left\{\left(v_{j}\right)_{j=0}^{N}:\left\|v-u^{(0)}\right\|_{1, \infty, \hat{\omega}_{h}} \leqslant r\right\} .
$$

Below, we use the following notation:

$$
\begin{aligned}
\|y\|_{0, \infty, \hat{\omega}_{h}} & =\max _{\xi \in \hat{\omega}_{h}}|y(\xi)|, \quad \hat{\omega}_{h}^{+}=\hat{\omega}_{h} \cup x_{N}, \quad\|y\|_{0, \infty, \hat{\omega}_{h}^{+}}=\max _{\xi \in \hat{\omega}_{h}^{+}}|y(\xi)|, \\
\|y\|_{1, \infty, \hat{\omega}_{h}} & =\max \left\{\|y\|_{0, \infty, \hat{\omega}_{h}},\left\|y_{\bar{x}}\right\|_{0, \infty, \hat{\omega}_{h}^{+}}\right\} \\
\|y\|_{0,1, \hat{\omega}_{h}} & =\sum_{\xi \in \hat{\omega}_{h}} \hbar(\xi)|y(\xi)|, \quad\|y\|_{0,1, \hat{\omega}_{h}^{+}}=\sum_{\xi \in \hat{\omega}_{h}^{+}} h(\xi)|y(\xi)| .
\end{aligned}
$$


Consider the initial value problems

$$
\begin{aligned}
\frac{d}{d x} w_{\alpha}^{j}(x, v) & =\frac{l_{\alpha}^{j}(x, v)}{k(x)}, \quad \frac{d}{d x} l_{\alpha}^{j}(x, v)=-f\left(x, Y_{\alpha}^{j}(x, v)\right), \quad x_{j-2+\alpha}<x<x_{j-1+\alpha}, \\
w_{\alpha}^{j}\left(x_{j+(-1)^{\alpha}}, v\right) & =l_{\alpha}^{j}\left(x_{j+(-1)^{\alpha}}, v\right)=0, \quad \alpha=1,2,
\end{aligned}
$$

where

$$
\begin{aligned}
Y_{\alpha}^{j}(x, v) & =\hat{v}(x)+w_{\alpha}^{j}(x, v)-\frac{V_{\alpha}^{j}(x)}{V_{\alpha}^{j}\left(x_{j}\right)} w_{\alpha}^{j}\left(x_{j}, v\right), x \in\left[x_{j-2+\alpha}, x_{j-1+\alpha}\right], \quad \alpha=1,2, \\
\hat{v}(x) & =\left[v\left(x_{j}\right) V_{1}^{j}(x)+v\left(x_{j-1}\right) V_{2}^{j-1}(x)\right] \cdot\left[V_{1}^{j}\left(x_{j}\right)\right]^{-1}, \quad x \in\left[x_{j-1}, x_{j}\right], \\
V_{1}^{j}(x) & =\int_{x_{j-1}}^{x} \frac{d t}{k(t)}, \quad V_{2}^{j}(x)=\int_{x}^{x_{j+1}} \frac{d t}{k(t)} .
\end{aligned}
$$

The following lemma is valid.

Lemma 3.1. Let conditions (2.1)-(2.3) be met. Then for $v_{j}=u\left(x_{j}\right), j=0,1, \ldots, N$ problems (3.1), (3.2) have a unique solution $w_{\alpha}^{j}(x, u), l_{\alpha}^{j}(x, u), \alpha=1,2$. Moreover, for condition (2.4), the solution of problem (1.1) can be represented as

$$
\begin{gathered}
u(x)=\hat{u}(x)+w_{\alpha}^{j}(x, u)-\frac{V_{\alpha}^{j}(x)}{V_{\alpha}^{j}\left(x_{j}\right)} w_{\alpha}^{j}\left(x_{j}, u\right), \\
x \in\left[x_{j-2+\alpha}, x_{j-1+\alpha}\right], \quad \alpha=1,2, \quad j=1,2, \ldots, N-1 .
\end{gathered}
$$

Proof. We write problems (3.1), (3.2) in an equivalent integral form

$$
w_{\alpha}^{j}(x, v)=\int_{x_{j+(-1)^{\alpha}}}^{x} \frac{l_{\alpha}^{j}(\xi, v)}{k(\xi)} d \xi=(-1)^{\alpha+1} \int_{x_{j+(-1)^{\alpha}}}^{x}\left[V_{\alpha}^{j}(\xi)-V_{\alpha}^{j}(x)\right] f\left(\xi, Y_{\alpha}^{j}(\xi, v)\right) d \xi, \quad \alpha=1,2
$$

or

$$
Y_{\alpha}^{j}(x, v)=\int_{x_{j-2+\alpha}}^{x_{j-1+\alpha}} G^{j-1+\alpha}(x, \xi) f\left(\xi, Y_{\alpha}^{j}(\xi, v)\right) d \xi+\hat{v}(x), x \in\left[x_{j-2+\alpha}, x_{j-1+\alpha}\right], \alpha=1,2,
$$

where

$$
G^{j-1+\alpha}(x, \xi)=\frac{1}{V_{1}^{j-1+\alpha}\left(x_{j-1+\alpha}\right)}\left\{\begin{array}{lr}
V_{1}^{j-1+\alpha}(x) V_{2}^{j-2+\alpha}(\xi), & x_{j-2+\alpha} \leqslant x \leqslant \xi \\
V_{2}^{j-2+\alpha}(x) V_{1}^{j-1+\alpha}(\xi), & \xi \leqslant x \leqslant x_{j-1+\alpha}
\end{array}\right.
$$

At $\alpha=1$ in view of (2.7), we have

$$
\begin{aligned}
\hat{u}(x)= & \frac{V_{1}^{j}(x)}{V_{1}^{j}\left(x_{j}\right)}\left[\int_{0}^{1} G\left(x_{j}, \xi\right) f(\xi, u(\xi)) d \xi+\frac{V_{2}\left(x_{j}\right)}{V_{1}(1)} \mu_{1}+\frac{V_{1}\left(x_{j}\right)}{V_{1}(1)} \mu_{2}\right] \\
& +\frac{V_{2}^{j-1}(x)}{V_{1}^{j}\left(x_{j}\right)}\left[\int_{0}^{1} G\left(x_{j-1}, \xi\right) f(\xi, u(\xi)) d \xi+\frac{V_{2}\left(x_{j-1}\right)}{V_{1}(1)} \mu_{1}+\frac{V_{1}\left(x_{j-1}\right)}{V_{1}(1)} \mu_{2}\right], \quad x \in\left[x_{j-1}, x_{j}\right] .
\end{aligned}
$$


Since

$$
\frac{V_{2}^{j-1}(x) V_{\alpha}\left(x_{j-1}\right)+V_{1}^{j}(x) V_{\alpha}\left(x_{j}\right)}{V_{1}^{j}\left(x_{j}\right) V_{1}(1)}=\frac{V_{\alpha}(x)}{V_{1}(1)}, \quad \alpha=1,2
$$

we obtain

$$
\begin{gathered}
\hat{u}(x)=\frac{V_{1}^{j}(x)}{V_{1}^{j}\left(x_{j}\right)} \int_{0}^{1} G\left(x_{j}, \xi\right) f(\xi, u(\xi)) d \xi+\frac{V_{2}^{j-1}(x)}{V_{1}^{j}\left(x_{j}\right)} \int_{0}^{1} G\left(x_{j-1}, \xi\right) f(\xi, u(\xi)) d \xi+u^{(0)}(x), \\
x \in\left[x_{j-1}, x_{j}\right] .
\end{gathered}
$$

Thus

$$
\begin{aligned}
Y_{1}^{j}(x, u)= & \frac{V_{1}^{j}(x)}{V_{1}^{j}\left(x_{j}\right)} \int_{0}^{1} G\left(x_{j}, \xi\right) f(\xi, u(\xi)) d \xi+\frac{V_{2}^{j-1}(x)}{V_{1}^{j}\left(x_{j}\right)} \int_{0}^{1} G\left(x_{j-1}, \xi\right) f(\xi, u(\xi)) d \xi \\
& +\int_{x_{j-1}}^{x_{j}} G^{j}(x, \xi) f\left(\xi, Y_{1}^{j}(\xi, u)\right) d \xi+u^{(0)}(x), \quad x \in\left[x_{j-1}, x_{j}\right] .
\end{aligned}
$$

By virtue of equality $Y_{2}^{j}(x, u)=Y_{1}^{j+1}(x, u)$, we have

$$
\begin{aligned}
Y_{2}^{j}(x, u)= & \left.\frac{V_{1}^{j+1}(x)}{V_{2}^{j}\left(x_{j}\right)} \int_{0}^{1} G\left(x_{j+1}, \xi\right) f(\xi, u(\xi)) d \xi+\frac{V_{2}^{j}(x)}{V_{2}^{j}\left(x_{j}\right)} \int_{0}^{1} G\left(x_{j}, \xi\right) f(\xi, u(\xi)) d \xi\right) \\
& +\int_{x_{j}}^{x_{j+1}} G^{j+1}(x, \xi) f\left(\xi, Y_{2}^{j}(\xi, u)\right) d \xi+u^{(0)}(x), \quad x \in\left[x_{j}, x_{j+1}\right] .
\end{aligned}
$$

Consequently, the existence and uniqueness of the solution to problems (3.1), (3.2) is equivalent to a similar problem for equations

$$
\begin{aligned}
U_{\alpha}^{j}(x)= & \Im_{\alpha}^{j}\left(x, u, U_{\alpha}^{j}\right)=\frac{V_{1}^{j-1+\alpha}(x)}{V_{\alpha}^{j}\left(x_{j}\right)} \int_{0}^{1} G\left(x_{j-1+\alpha}, \xi\right) f(\xi, u(\xi)) d \xi \\
& +\frac{V_{2}^{j-2+\alpha}(x)}{V_{\alpha}^{j}\left(x_{j}\right)} \int_{0}^{1} G\left(x_{j-2+\alpha}, \xi\right) f(\xi, u(\xi)) d \xi \\
& +\int_{x_{j-2+\alpha}}^{x_{j-1+\alpha}} G^{j-1+\alpha}(x, \xi) f\left(\xi, U_{\alpha}^{j}(\xi)\right) d \xi+u^{(0)}(x), \quad x \in\left[x_{j-2+\alpha}, x_{j-1+\alpha}\right], \quad \alpha=1,2 .
\end{aligned}
$$


We show that the operator $\Im_{1}\left(x, u, U_{1}^{j}\right)$ maps the set $\Omega\left(\left[x_{j-1}, x_{j}\right], r\right)$ onto itself.

$$
\begin{aligned}
& \left|\Im_{1}\left(x, u, U_{1}^{j}\right)-u^{(0)}(x)\right| \\
\leqslant & K\left\{\frac{V_{2}^{j-1}(x)}{V_{1}^{j}\left(x_{j}\right)} \int_{0}^{1} G\left(x_{j-1}, \xi\right) d \xi+\frac{V_{1}^{j}(x)}{V_{1}^{j}\left(x_{j}\right)} \int_{0}^{1} G\left(x_{j}, \xi\right) d \xi+\int_{x_{j-1}}^{x_{j}} G^{j}(x, \xi) d \xi\right\} \\
= & K \frac{V_{2}^{j-1}(x)}{V(1) V_{1}^{j}\left(x_{j}\right)}\left[V_{2}\left(x_{j-1}\right) \int_{0}^{x} V_{1}(\xi) d \xi+V_{1}\left(x_{j-1}\right) \int_{x}^{1} V_{2}(\xi) d \xi\right] \\
& +K \frac{V_{1}^{j}(x)}{V(1) V_{1}^{j}\left(x_{j}\right)}\left[V_{2}\left(x_{j}\right) \int_{0}^{x} V_{1}(\xi) d \xi+V_{1}\left(x_{j}\right) \int_{x}^{1} V_{2}(\xi) d \xi\right] \\
& +K \frac{V_{2}^{j-1}(x)}{V_{1}^{j}\left(x_{j}\right)}\left\{\frac{1}{V(1)} \int_{x_{j-1}}^{x}\left[V_{1}\left(x_{j-1}\right) V_{2}(\xi)-V_{2}\left(x_{j-1}\right) V_{1}(\xi)\right] d \xi+\int_{x_{j-1}}^{x} V_{1}^{j}(\xi) d \xi\right\} \\
& -K \frac{V_{1}^{j}(x)}{V_{1}^{j}\left(x_{j}\right)}\left\{\frac{1}{V(1)} \int_{x}^{x_{j}}\left[V_{1}\left(x_{j}\right) V_{2}(\xi)-V_{2}\left(x_{j}\right) V_{1}(\xi)\right] d \xi-\int_{x}^{V_{2}^{j-1}(\xi) d \xi}\right\} .
\end{aligned}
$$

Taking identity (3.5) and

$$
\frac{V_{1}\left(x_{j-1}\right) V_{2}(\xi)-V_{2}\left(x_{j-1}\right) V_{1}(\xi)}{V_{1}(1)}=-V_{1}^{j}(\xi), \frac{V_{1}\left(x_{j}\right) V_{2}(\xi)-V_{2}\left(x_{j}\right) V_{1}(\xi)}{V_{1}(1)}=V_{2}^{j-1}(\xi),
$$

we obtain

$$
\left|\Im_{1}\left(x, u, U_{1}^{j}\right)-u^{(0)}(x)\right| \leqslant K \frac{V_{2}(x)}{V_{1}(1)} \int_{0}^{x} V_{1}(\xi) d \xi+K \frac{V_{1}(x)}{V_{1}(1)} \int_{x}^{1} V_{2}(\xi) d \xi=K \int_{0}^{1} G(x, \xi) d \xi .
$$

Consequently,

$$
\left\|\Im_{1}\left(x, u, U_{1}^{j}\right)-u^{(0)}\right\|_{1, \infty,\left(x_{j-1}, x_{j}\right)} \leqslant \frac{K}{c_{1}}=r,
$$

i.e., the operator $\Im_{1}\left(x, u, U_{1}^{j}\right)$ maps the set $\Omega\left(\left[x_{j-1}, x_{j}\right], r\right)$ onto itself.

Analogously, it is possible to show that if $U_{2}^{j}(x) \in \Omega\left(\left[x_{j}, x_{j+1}\right], r\right)$, then

$$
\left\|\Im_{2}\left(x, u, U_{2}^{j}\right)-u^{(0)}\right\|_{1, \infty,\left(x_{j}, x_{j+1}\right)} \leqslant r .
$$

For $\forall U_{\alpha}^{j}(x), \tilde{U}_{\alpha}^{j}(x) \in \Omega\left(\left[x_{j-2+\alpha}, x_{j-1+\alpha}\right], r\right)$ in view of

$$
G^{j-1+\alpha}(x, \xi) \leqslant \frac{h_{j-1+\alpha}}{c_{1}}, \quad\left|\frac{\partial G^{j-1+\alpha}(x, \xi)}{\partial x}\right| \leqslant \frac{1}{c_{1}},
$$

we have

$$
\begin{aligned}
\left\|\Im_{\alpha}\left(x, u, U_{\alpha}^{j}\right)-\Im_{\alpha}\left(x, u, \tilde{U}_{\alpha}^{j}\right)\right\|_{1, \infty,\left(x_{j-2+\alpha}, x_{j-1+\alpha}\right)} & \leqslant \frac{1}{c_{1}} \int_{x_{j-2+\alpha}}^{x_{j-1+\alpha}}\left|f\left(\xi, U_{\alpha}^{j}(\xi, u)\right)-f\left(\xi, \tilde{U}_{\alpha}^{j}(\xi, u)\right)\right| d \xi \\
& \leqslant \frac{h_{j-1+\alpha} L}{c_{1}}\left\|U_{\alpha}^{j}-\tilde{U}_{\alpha}^{j}\right\|_{1, \infty,\left(x_{j-2+\alpha}, x_{j-1+\alpha}\right)} \\
& \leqslant q\left\|U_{\alpha}^{j}-\tilde{U}_{\alpha}^{j}\right\|_{1, \infty,\left(x_{j-2+\alpha}, x_{j-1+\alpha}\right)},
\end{aligned}
$$


where $q=L / c_{1}<1$.

Thus, for operators (3.6) in the area $\Omega\left(\left[x_{j-2+\alpha}, x_{j-1+\alpha}\right], r\right)$, all conditions of the principle of contraction mapping are fulfilled and, hence, problems (3.1), (3.2) have a unique solution.

Since

$$
\frac{d Y_{\alpha}^{j}(x, u)}{d x}=\frac{1}{k(x)}\left[\frac{h_{j-1+\alpha} u_{\bar{x}, j-1+\alpha}+(-1)^{\alpha} w_{\alpha}^{j}\left(x_{j}, u\right)}{V_{\alpha}^{j}\left(x_{j}\right)}+l_{\alpha}^{j}(x, u)\right]
$$

and

$$
\frac{d}{d x}\left(k(x) \frac{d Y_{\alpha}^{j}(x, u)}{d x}\right)=\frac{d l_{\alpha}^{j}(x, u)}{d x}=-f\left(x, Y_{\alpha}^{j}(x, u)\right),
$$

we conclude that the functions $Y_{\alpha}^{j}(x, u), \alpha=1,2$ are defined as solutions of the problems

$$
\begin{gathered}
\frac{d}{d x}\left(k(x) \frac{d Y_{\alpha}^{j}(x, u)}{d x}\right)=-f\left(x, Y_{\alpha}^{j}(x, u)\right), \quad x \in\left(x_{j-2+\alpha}, x_{j-1+\alpha}\right), \\
Y_{\alpha}^{j}\left(x_{j-2+\alpha}, u\right)=u\left(x_{j-2+\alpha}\right), \quad Y_{\alpha}^{j}\left(x_{j-1+\alpha}, u\right)=u\left(x_{j-1+\alpha}\right), \quad \alpha=1,2, \quad j=1,2, \ldots, N-1 .
\end{gathered}
$$

Theorem 3.1. Let the conditions of Theorem 2.1 be met. Then for problem (1.1) there exists an ETDS of the form

$$
\left(a u_{\bar{x}}\right)_{\hat{x}}=-\hat{T}^{x}(f(\xi, u(\xi))), x \in \hat{\omega}_{h}, u(0)=\mu_{1}, u(1)=\mu_{2},
$$

where

$$
\begin{aligned}
& u_{\bar{x}, j}=\frac{u_{j}-u_{j-1}}{h_{j}}, \quad u_{\hat{x}, j}=\frac{u_{j+1}-u_{j}}{\hbar_{j}}, \quad \hbar_{j}=\frac{h_{j}+h_{j+1}}{2}, \\
& a\left(x_{j}\right)=\left[\frac{1}{h_{j}} V_{1}^{j}\left(x_{j}\right)\right]^{-1}, \\
& \hat{T}^{x_{j}}(w(\xi))=\left[\hbar_{j} V_{1}^{j}\left(x_{j}\right)\right]^{-1} \int_{x_{j-1}}^{x_{j}} V_{1}^{j}(\xi) w(\xi) d \xi+\left[\hbar_{j} V_{2}^{j}\left(x_{j}\right)\right]^{-1} \int_{x_{j}}^{x_{j+1}} V_{2}^{j}(\xi) w(\xi) d \xi,
\end{aligned}
$$

and the function $u(\xi)$ on the right-hand side of (3.7) is defined by formula (3.3) and only depends on $\left(u_{j}\right)_{j=0}^{N}$.

Proof. The operator $\hat{T}^{x_{j}}$ applied to both sides of (1.1) produces (see, e.g., [8])

$$
\hat{T}^{x_{j}}\left(\frac{d}{d \xi}\left(k(\xi) \frac{d u(\xi)}{d \xi}\right)\right)=-\hat{T}^{x_{j}}(f(\xi, u(\xi))),
$$

where

$$
\hat{T}^{x_{j}}\left(\frac{d}{d \xi}\left(k(\xi) \frac{d u(\xi)}{d \xi}\right)\right)=\left(a u_{\bar{x}}\right)_{\hat{x}, j} .
$$

Combining this with (3.3), we prove the statement.

To prove the uniqueness of the solution to ETDS (3.7) the following lemma is necessary.

Lemma 3.2. Let condition (2.1) be satisfied and a constant $\Delta>0$ such that conditions (2.2) and (2.3) hold in $\Omega([0,1], r+\Delta)$ exists. Then $\exists h_{0}>0$ such that for $|h| \leqslant h_{0}(|h|=$ $\left.\max _{1 \leqslant i \leqslant N} h_{i}\right)$ and $\forall\left(v_{j}\right)_{j=0}^{N} \in \Omega\left(\hat{\bar{\omega}}_{h}, r\right)$ problems (3.1), (3.2) have a unique solution. 
Proof. Since, by virtue of (3.4) problems (3.1), (3.2) are equivalent to the operator equations

$$
\begin{gathered}
U_{\alpha}^{j}(x)=\Re_{\alpha}^{j}\left(x, v, U_{\alpha}^{j}\right)=\int_{x_{j-2+\alpha}}^{x_{j-1+\alpha}} G^{j-1+\alpha}(x, \xi) f\left(\xi, U_{\alpha}^{j}(\xi)\right) d \xi+\hat{v}(x), \\
x \in\left[x_{j-2+\alpha}, x_{j-1+\alpha}\right], \quad \alpha=1,2,
\end{gathered}
$$

we examine the properties of the operators $\Re_{\alpha}^{j}\left(x, v, U_{\alpha}^{j}\right), \alpha=1,2$. Taking into account equality (3.5), we obtain $u^{(0)}(x)=\hat{u}^{(0)}(x)$. Let $U_{\alpha}^{j}(x) \in \Omega\left(\left[x_{j-2+\alpha}, x_{j-1+\alpha}\right], r+\Delta\right)$, then

$$
\begin{aligned}
&\left\|\Re_{\alpha}^{j}\left(x, v, U_{\alpha}^{j}\right)-u^{(0)}\right\|_{1, \infty,\left(x_{j-2+\alpha}, x_{j-1+\alpha}\right)} \leqslant\left\|\hat{v}-\hat{u}^{(0)}\right\|_{1, \infty, \hat{\omega}_{h}}+\frac{1}{c_{1}} \int_{x_{j-2+\alpha}}^{x_{j-1+\alpha}}\left|f\left(\xi, U_{\alpha}^{j}(\xi)\right)\right| d \xi \\
& \leqslant\left\|\hat{v}-\hat{u}^{(0)}\right\|_{1, \infty, \hat{\omega}_{h}}+\frac{|h| K}{c_{1}} \leqslant r+r|h| \leqslant r+\Delta, \\
& \forall\left(v_{j}\right)_{j=0}^{N} \in \Omega\left(\hat{\bar{\omega}}_{h}, r\right),
\end{aligned}
$$

i.e., the operators $\Re_{\alpha}^{j}\left(x, v, U_{\alpha}^{j}\right), \alpha=1,2$ for $|h| \leqslant h_{0}$ and $\forall\left(v_{j}\right)_{j=0}^{N} \in \Omega\left(\hat{\bar{\omega}}_{h}, r\right)$ map the set $\Omega\left(\left[x_{j-2+\alpha}, x_{j-1+\alpha}\right], r+\Delta\right)$ onto themselves.

Moreover, for $\forall U_{\alpha}^{j}(x), \tilde{U}_{\alpha}^{j}(x) \in \Omega\left(\left[x_{j-2+\alpha}, x_{j-1+\alpha}\right], r+\Delta\right)$ we have the estimate

$$
\begin{aligned}
\left\|\Re_{\alpha}^{j}\left(x, v, U_{\alpha}^{j}\right)-\Re_{\alpha}^{j}\left(x, v, \tilde{U}_{\alpha}^{j}\right)\right\|_{1, \infty,\left(x_{j-2+\alpha}, x_{j-1+\alpha}\right)} & \leqslant \frac{1}{c_{1}} \int_{x_{j-2+\alpha}}^{x_{j-1+\alpha}}\left|f\left(\xi, U_{\alpha}^{j}(\xi)\right)-f\left(\xi, \tilde{U}_{\alpha}^{j}(\xi)\right)\right| d \xi \\
& \leqslant \frac{h_{j-1+\alpha} L}{c_{1}}\left\|U_{\alpha}^{j}-\tilde{U}_{\alpha}^{j}\right\|_{1, \infty,\left(x_{j-2+\alpha}, x_{j-1+\alpha}\right)} \\
& \leqslant q\left\|U_{\alpha}^{j}-\tilde{U}_{\alpha}^{j}\right\|_{1, \infty,\left(x_{j-2+\alpha}, x_{j-1+\alpha}\right)} .
\end{aligned}
$$

Consequently, the operators $\Re_{\alpha}^{j}\left(x, v, U_{\alpha}^{j}\right), \quad \alpha=1,2$ are a contraction on $\Omega\left(\left[x_{j-2+\alpha}, x_{j-1+\alpha}\right], r+\Delta\right)$ and problems (3.1), (3.2) have a unique solution for all $\left(v_{j}\right)_{j=0}^{N} \in$ $\Omega\left(\hat{\bar{\omega}}_{h}, r\right)$.

The existence of a solution to the nonlinear ETDS (3.7) is proved in Theorem 3.1. The following lemma establishes the uniqueness.

Lemma 3.3. Let the conditions of Theorem 2.1 be met. Then $\exists h_{0}>0$ such that for $|h| \leqslant h_{0}$ and $\forall\left(u_{j}\right)_{j=0}^{N} \in \Omega\left(\hat{\bar{\omega}}_{h}, r\right) \operatorname{ETDS}$ (3.7) has a unique solution, which can be obtained by the method of successive approximations:

$$
\begin{aligned}
\left(a u_{\bar{x}}^{(n)}\right)_{\hat{x}} & =-\hat{T}^{x}\left(f\left(\xi, u^{(n-1)}(\xi)\right)\right), \quad x \in \hat{\omega}_{h}, \quad u^{(n)}(0)=\mu_{1}, \quad u^{(n)}(1)=\mu_{2}, \\
u^{(n)}(x) & =\hat{u}^{(n)}(x)+w_{\alpha}^{j}\left(x, u^{(n)}\right)-\frac{V_{\alpha}^{j}(x)}{V_{\alpha}^{j}\left(x_{j}\right)} w_{\alpha}^{j}\left(x_{j}, u^{(n)}\right), \quad x \in\left[x_{j-2+\alpha}, x_{j-1+\alpha}\right], \quad \alpha=1,2, \\
\hat{u}^{(n)}(x) & =\left[u^{(n)}\left(x_{j}\right) V_{1}^{j}(x)+u^{(n)}\left(x_{j-1}\right) V_{2}^{j-1}(x)\right] \cdot\left[V_{1}^{j}\left(x_{j}\right)\right]^{-1}, \quad x \in\left[x_{j-1}, x_{j}\right], \\
u^{(0)}(x) & =\frac{V_{2}(x)}{V_{1}(1)} \mu_{1}+\frac{V_{1}(x)}{V_{1}(1)} \mu_{2}
\end{aligned}
$$


with the error estimate

$$
\left\|u^{(n)}-u\right\|_{1, \infty, \hat{\omega}_{h}}^{*}=\max \left\{\left\|u^{(n)}-u\right\|_{0, \infty, \hat{\omega}_{h}},\left\|k \frac{d u^{(n)}}{d x}-k \frac{d u}{d x}\right\|_{0, \infty, \hat{\omega}_{h}}\right\} \leqslant M q_{1}^{n},
$$

where $q_{1}=q+M_{1}|h|<1, M, M_{1}$ are constants.

Proof. Let us use the principle of contraction mappings. Since the Green function $G^{h}(x, \xi)$ of problem (3.7) coincides with the projection of the Green function $G(x, \xi)$ from (2.8) of problem (1.1) on the grid $\hat{\omega}_{h}$, ETDS (3.7) can be written in the form

$$
u(x)=\Re_{h}\left(x,\left(u_{j}\right)_{j=0}^{N}\right)=\sum_{\xi \in \hat{\omega}_{h}} \hbar(\xi) G(x, \xi) \hat{T}^{\xi}(f(\eta, u(\eta)))+u^{(0)}(x), \forall x \in \hat{\bar{\omega}}_{h},
$$

Examine the properties of the operator $\Re_{h}\left(x,\left(u_{j}\right)_{j=0}^{N}\right)$.

Note that the operator $\hat{T}^{\xi}$ possesses the following property: $\forall Q(\xi) \in \stackrel{\circ}{H}_{h}$ and $w(\eta) \in$ $L_{1}(0,1)\left(\stackrel{\circ}{H}_{h}\right.$ is the space of the grid functions $v(x), x \in \hat{\bar{\omega}}_{h}$ such that $\left.v(0)=v(1)=0\right)$ satisfy the identity

$$
\begin{aligned}
\sum_{\xi \in \hat{\omega}_{h}} \hbar(\xi) \hat{T}^{\xi}(w(\eta)) Q(\xi)= & \sum_{i=1}^{N-1} \hbar_{j} Q\left(x_{i}\right) \frac{1}{\hbar_{j} V_{1}^{i}\left(x_{i}\right)} \int_{x_{i-1}}^{x_{i}} V_{1}^{i}(\eta) w(\eta) d \eta \\
& +\sum_{i=1}^{x_{i+1}} \hbar_{i} Q\left(x_{i}\right) \frac{1}{\hbar_{i} V_{2}^{i}\left(x_{i}\right)} \int_{x_{i}}^{N-1} V_{2}^{i}(\eta) w(\eta) d \eta \\
= & \sum_{i=1}^{N} \int_{x_{i-1}}^{x_{i}}\left\{Q\left(x_{i}\right) \frac{V_{1}^{i}(\eta)}{V_{1}^{i}\left(x_{i}\right)}+Q\left(x_{i-1}\right) \frac{V_{2}^{i-1}(\eta)}{V_{1}^{i}\left(x_{i}\right)}\right\} w(\eta) d \eta
\end{aligned}
$$

By virtue of (3.11), we have

$$
\begin{aligned}
\Re_{h}\left(x,\left(u_{j}\right)_{j=0}^{N}\right)= & \sum_{i=1}^{N} \int_{x_{i-1}}^{x_{i}}\left\{G\left(x, x_{i}\right) \frac{V_{1}^{i}(\eta)}{V_{1}^{i}\left(x_{i}\right)}\right. \\
& \left.+G\left(x, x_{i-1}\right) \frac{V_{2}^{i-1}(\eta)}{V_{1}^{i}\left(x_{i}\right)}\right\} f(\eta, u(\eta)) d \eta+u^{(0)}(x), \quad x \in \hat{\omega}_{h},
\end{aligned}
$$

where

$$
u(\eta)=\hat{u}(\eta)+w_{1}^{i}(\eta, u)-\frac{V_{1}^{i}(\eta)}{V_{1}^{i}\left(x_{i}\right)} w_{1}^{i}\left(x_{i}, u\right), \quad \eta \in\left[x_{i-1}, x_{i}\right] .
$$

We show that the operator (3.12) maps the set $\Omega\left(\hat{\bar{\omega}}_{h}, r\right)$ onto itself. Let $\left(v_{j}\right)_{j=0}^{N} \in \Omega\left(\hat{\bar{\omega}}_{h}, r\right)$, then $v(x)=\hat{v}(x)+w_{1}^{j}(x, v)-\frac{V_{1}^{j}(x)}{V_{1}^{j}\left(x_{j}\right)} w_{1}^{j}\left(x_{j}, v\right) \in \Omega\left(\left[x_{j-1}, x_{j}\right], r+\Delta\right)$ (see the proof of Lemma 3.1). Taking into account the relations

$$
G(x, \xi) \leqslant \frac{1}{c_{1}}, \quad\left|G_{\bar{x}}(x, \xi)\right| \leqslant \frac{1}{c_{1}},
$$


condition (2.2), and the equality $V_{1}^{j}(x)+V_{2}^{j-1}(x)=V_{1}^{j}\left(x_{j}\right)$, we have

$$
\left\|\Re_{h}\left(x,\left(v_{j}\right)_{j=0}^{N}\right)-u^{(0)}(x)\right\|_{1, \infty, \hat{\omega}_{h}} \leqslant \frac{K}{c_{1}} \sum_{i=1}^{N} h_{i}=r, \quad \forall\left(v_{j}\right)_{j=0}^{N} \in \Omega\left(\hat{\bar{\omega}}_{h}, r\right) .
$$

Moreover,

$$
\begin{aligned}
\left\|\Re_{h}\left(x,\left(u_{j}\right)_{j=0}^{N}\right)-\Re_{h}\left(x,\left(v_{j}\right)_{j=0}^{N}\right)\right\|_{1, \infty, \hat{\omega}_{h}} & \leqslant \frac{1}{c_{1}} \sum_{i=1}^{N} \int_{x_{i-1}}^{x_{i}}|f(\xi, u(\xi))-f(\xi, v(\xi))| d \xi \\
& \leqslant \frac{L}{c_{1}} \max _{1 \leqslant j \leqslant N}\|u-v\|_{0, \infty,\left(x_{j-1}, x_{j}\right)} \sum_{i=1}^{N} h_{i} \\
& =q\|u-v\|_{0, \infty,(0,1)}, \\
\forall\left(u_{j}\right)_{j=0}^{N},\left(v_{j}\right)_{j=0}^{N} \in \Omega\left(\hat{\bar{\omega}}_{h}, r\right) . &
\end{aligned}
$$

Let us show that

$$
\|u-v\|_{0, \infty,(0,1)} \leqslant(1+M|h|)\|u-v\|_{0, \infty, \hat{\omega}_{h}} .
$$

Upon the substitution

$$
\begin{aligned}
& u(x)=\hat{u}(x)+\tilde{u}(x), \quad x_{j-1} \leqslant x \leqslant x_{j}, \\
& \tilde{u}(x)=w_{1}^{j}(x, u)-\frac{V_{1}^{j}(x)}{V_{1}^{j}\left(x_{j}\right)} w_{1}^{j}\left(x_{j}, u\right)
\end{aligned}
$$

the problem

$$
\begin{aligned}
\frac{d}{d x}\left[k(x) \frac{d u}{d x}\right] & =-f(x, u(x)), \quad x \in\left(x_{j-1}, x_{j}\right), \\
u\left(x_{j-1}\right) & =u_{j-1}, \quad u\left(x_{j}\right)=u_{j}
\end{aligned}
$$

is reduced to the form

$$
\begin{aligned}
\frac{d}{d x}\left[k(x) \frac{d \tilde{u}}{d x}\right] & =-f(x, \hat{u}(x)+\tilde{u}(x)), \quad x \in\left(x_{j-1}, x_{j}\right), \\
\tilde{u}\left(x_{j-1}\right) & =\tilde{u}\left(x_{j}\right)=0 .
\end{aligned}
$$

Then

$$
\tilde{u}(x)=\int_{x_{j-1}}^{x_{j}} G^{j}(x, \xi) f(\xi, \hat{u}(\xi)+\tilde{u}(\xi)) d \xi, \quad x_{j-1} \leqslant x \leqslant x_{j}
$$

where

$$
G^{j}(x, \xi)=\frac{1}{V_{1}^{j}\left(x_{j}\right)} \begin{cases}V_{1}^{j}(x) V_{2}^{j-1}(\xi), & x_{j-1} \leqslant x \leqslant \xi \\ V_{2}^{j-1}(x) V_{1}^{j}(\xi), & \xi \leqslant x \leqslant x_{j} .\end{cases}
$$

By virtue of the Lipschitz condition

$$
\begin{aligned}
\|\tilde{u}-\tilde{v}\|_{1, \infty,\left(x_{j-1}, x_{j}\right)} & \leqslant \frac{1}{c_{1}} \int_{x_{j-1}}^{x_{j}}|f(\xi, \hat{u}(\xi)+\tilde{u}(\xi))-f(\xi, \hat{v}(\xi)+\tilde{v}(\xi))| d \xi \\
& \leqslant \frac{|h| L}{c_{1}}\left[\|\hat{u}-\hat{v}\|_{0, \infty,\left(x_{j-1}, x_{j}\right)}+\|\tilde{u}-\tilde{v}\|_{\left.0, \infty, x_{j-1}, x_{j}\right)}\right] \\
& \leqslant|h| q\|u-v\|_{0, \infty, \hat{\omega}_{h}}+|h| q\|\tilde{u}-\tilde{v}\|_{1, \infty,\left(x_{j-1}, x_{j}\right)} .
\end{aligned}
$$


It follows from this that

$$
\|\tilde{u}-\tilde{v}\|_{1, \infty,\left(x_{j-1}, x_{j}\right)} \leqslant \frac{|h| q}{1-|h| q}\|u-v\|_{0, \infty, \omega_{h}} \leqslant|h| M_{2}\|u-v\|_{0, \infty, \omega_{h}} .
$$

Then (3.13) follows from

$$
\begin{aligned}
\|u-v\|_{0, \infty,\left(x_{j-1}, x_{j}\right)} & \leqslant\|\hat{u}-\hat{v}\|_{0, \infty,\left(x_{j-1}, x_{j}\right)}+\|\tilde{u}-\tilde{v}\|_{0, \infty,\left(x_{j-1}, x_{j}\right)} \\
& \leqslant\|u-v\|_{0, \infty, \hat{\omega}_{h}}+|h| M_{2}\|u-v\|_{0, \infty, \hat{\omega}_{h}} .
\end{aligned}
$$

In view of inequality (3.13), we obtain

$$
\left\|\Re_{h}\left(x,\left(u_{j}\right)_{j=0}^{N}\right)-\Re_{h}\left(x,\left(v_{j}\right)_{j=0}^{N}\right)\right\|_{1, \infty, \hat{\omega}_{h}} \leqslant\left(q+M_{1}|h|\right)\|u-v\|_{1, \infty, \hat{\omega}_{h}}=q_{1}\|u-v\|_{1, \infty, \hat{\omega}_{h}} .
$$

By virtue of (2.4), we have $q_{1}=q+M_{1}|h|<1$ for a sufficiently small $h_{0}$ and operator (3.12) $\forall\left(u_{j}\right)_{j=0}^{N},\left(v_{j}\right)_{j=0}^{N} \in \Omega\left(\hat{\bar{\omega}}_{h}, r\right)$ is a contraction.

Thus, according to the principle of contractive mappings for a sufficiently small $h_{0}$ ETDS (3.7) has a unique solution, which can be obtained by the method of successive approximations (3.9) with the error estimate

$$
\left\|u^{(n)}-u\right\|_{1, \infty, \hat{\omega}_{h}} \leqslant \frac{q_{1}^{n}}{1-q_{1}} r .
$$

Moreover, using (3.14), (3.15)

$$
\begin{aligned}
\left\|k \frac{d u^{(n)}}{d x}-k \frac{d u}{d x}\right\|_{0, \infty, \hat{\omega}_{h}} & \leqslant\left\|k \frac{d \hat{u}^{(n)}}{d x}-k \frac{d \hat{u}}{d x}\right\|_{0, \infty, \hat{\omega}_{h}}+\left\|k \frac{d \tilde{u}^{(n)}}{d x}-k \frac{d \tilde{u}}{d x}\right\|_{0, \infty, \hat{\omega}_{h}} \\
& \leqslant|h| M_{1}\left\|u_{\bar{x}}^{(n)}-u_{\bar{x}}\right\|_{0, \infty, \hat{\omega}_{h}^{+}}+M_{2}\left\|\tilde{u}^{(n)}-\tilde{u}\right\|_{1, \infty,(0,1)} \\
& \leqslant M_{3}\left\|u^{(n)}-u\right\|_{1, \infty, \hat{\omega}_{h}} \leqslant M q_{1}^{n} .
\end{aligned}
$$

Hence, estimate (3.10) is valid.

\section{Algorithmic implementation of the exact three-point difference schemes}

First, we take into account that, because of

$$
(-1)^{\alpha+1} \int_{x_{j+(-1)^{\alpha}}}^{x_{j}} V_{\alpha}^{j}(\xi) f(\xi, u) d \xi=(-1)^{\alpha} V_{\alpha}^{j}\left(x_{j}\right) l_{\alpha}^{j}\left(x_{j}, u\right)+w_{\alpha}^{j}\left(x_{j}, u\right), \quad \alpha=1,2,
$$

we have

$$
\varphi\left(x_{j}, u\right)=\hat{T}^{x_{j}}(f(\xi, u(\xi)))=\hbar_{j}^{-1} \sum_{\alpha=1}^{2}(-1)^{\alpha}\left[l_{\alpha}^{j}\left(x_{j}, u\right)+(-1)^{\alpha} \frac{w_{\alpha}^{j}\left(x_{j}, u\right)}{V_{\alpha}^{j}(x)}\right] .
$$

Therefore, to construct the ETDS defined by (3.7), (3.8), and (4.1) $\forall x_{j} \in \hat{\omega}_{h}$, it is necessary to solve two initial value problems $(3.1)$ : one $(\alpha=1)$ in the forward direction on the 
closed interval $\left[x_{j-1}, x_{j}\right]$, and the other $(\alpha=2)$ in the backward direction on $\left[x_{j}, x_{j+1}\right]$; both problems have smooth coefficients. To solve them numerically, we apply the one-step Taylor series expansion method of the $\bar{m}$ th order of accuracy.

Note that the functions $w_{\alpha}^{j}(x, u), l_{\alpha}^{j}(x, u), \alpha=1,2$, which are the solutions of systems (3.1), depend on the parameters $b_{\alpha} \equiv b_{\alpha}^{j}(u)=w_{\alpha}^{j}\left(x_{j}, u\right)$, i.e., $w_{\alpha}^{j}(x, u) \equiv w_{\alpha}^{j}\left(x, u, b_{\alpha}\right)$, $l_{\alpha}^{j}(x, u) \equiv l_{\alpha}^{j}\left(x, u, b_{\alpha}\right), \alpha=1,2$.

The algorithm for the solution of problems (3.1) works as follows.

1. By successive differentiation (3.1) we find the derivatives

$$
\begin{aligned}
\frac{d^{2} w_{\alpha}^{j}\left(x, u, b_{\alpha}\right)}{d x^{2}}= & \frac{d}{d x}\left[\frac{1}{k(x)}\right] \cdot l_{\alpha}^{j}\left(x, u, b_{\alpha}\right)+\frac{1}{k(x)} \frac{d l_{\alpha}^{j}\left(x, u, b_{\alpha}\right)}{d x}, \\
\frac{d^{2} l_{\alpha}^{j}\left(x, u, b_{\alpha}\right)}{d x^{2}}= & -\frac{\partial f}{\partial x}-\frac{\partial f}{\partial u} \frac{d u}{d x} \\
\frac{d^{3} w_{\alpha}^{j}\left(x, u, b_{\alpha}\right)}{d x^{3}}= & \frac{d^{2}}{d x^{2}}\left[\frac{1}{k(x)}\right] \cdot l_{\alpha}^{j}\left(x, u, b_{\alpha}\right) \\
& +2 \frac{d}{d x}\left[\frac{1}{k(x)}\right] \cdot \frac{d l_{\alpha}^{j}\left(x, u, b_{\alpha}\right)}{d x}+\frac{1}{k(x)} \frac{d^{2} l_{\alpha}^{j}\left(x, u, b_{\alpha}\right)}{d x^{2}}, \\
\frac{d^{3} l_{\alpha}^{j}\left(x, u, b_{\alpha}\right)}{d x^{3}}= & -\frac{\partial^{2} f}{\partial x^{2}}-2 \frac{\partial^{2} f}{\partial x \partial u} \frac{d u}{d x}-\frac{\partial^{2} f}{\partial u^{2}}\left(\frac{d u}{d x}\right)^{2}-\frac{\partial f}{\partial u} \frac{d^{2} u}{d x^{2}}, \\
\frac{d^{4} w_{\alpha}^{j}\left(x, u, b_{\alpha}\right)}{d x^{4}}= & \frac{d^{3}}{d x^{3}}\left[\frac{1}{k(x)}\right] \cdot l_{\alpha}^{j}\left(x, u, b_{\alpha}\right)+3 \frac{d^{2}}{d x^{2}}\left[\frac{1}{k(x)}\right] \cdot \frac{d l_{\alpha}^{j}\left(x, u, b_{\alpha}\right)}{d x} \\
& +3 \frac{d}{d x}\left[\frac{1}{k(x)}\right] \frac{d^{2} l_{\alpha}^{j}\left(x, u, b_{\alpha}\right)}{d x^{2}}+\frac{1}{k(x)} \frac{d^{3} l_{\alpha}^{j}\left(x, u, b_{\alpha}\right)}{d x^{3}}, \\
& -3 \frac{\partial^{2} f}{\partial x \partial u} \frac{d^{2} u}{\partial x^{2}}-3 \frac{\partial^{2} f}{\partial u^{2}} \frac{d u}{d x} \frac{d^{3} u}{d x^{2}}-\frac{\partial^{3} f}{\partial u^{3}}\left(\frac{d u}{d x}\right)^{3}-\frac{\partial f}{\partial u} \frac{d u}{d x^{3}},
\end{aligned}
$$

where

$$
\begin{gathered}
\frac{d^{p} u}{d x^{p}}=\frac{d^{p-1}}{d x^{p-1}}\left[\frac{1}{k(x)}\right] \frac{h_{j-1+\alpha} u_{\bar{x}, j-1+\alpha}+(-1)^{\alpha} b_{\alpha}}{V_{\alpha}^{j}\left(x_{j}\right)}+\frac{d^{p} w_{\alpha}^{j}(x, u)}{d x^{p}}, \\
p=1,2, \ldots, \quad x \in\left[x_{j-2+\alpha}, x_{j-1+\alpha}\right], \quad \alpha=1,2 .
\end{gathered}
$$

2. Evaluate the approximate values of parameters $b_{\alpha}, \alpha=1,2$ from the formulas

$$
\begin{aligned}
b_{\alpha}^{(s-1)}= & w_{\alpha}^{(s-1) j}\left(x_{j}, u\right)=-\frac{h_{j-1+\alpha}^{2}}{2} \frac{f_{j+(-1)^{\alpha}}}{k_{j+(-1)^{\alpha}}} \\
& +\sum_{p=3}^{s-1} \frac{\left[(-1)^{\alpha+1} h_{j-1+\alpha}\right]^{p}}{p !} \frac{d^{p} w_{\alpha}^{j}\left(x_{\left.j+(-1)^{\alpha}, u, b_{\alpha}^{(s-2)}\right)}\right.}{d x^{p}}, \quad s=3,4, \ldots, \bar{m}, \quad \sum_{p=3}^{2}=0,
\end{aligned}
$$


3. Calculate now the approximate solutions of problems (3.1)

$$
\begin{aligned}
w_{\alpha}^{(\bar{m}) j}\left(x_{j}, u\right)= & -\frac{h_{j-1+\alpha}^{2}}{2} \frac{f_{j+(-1)^{\alpha}}}{k_{j+(-1)^{\alpha}}} \\
& +\sum_{p=3}^{\bar{m}} \frac{\left[(-1)^{\alpha+1} h_{j-1+\alpha}\right]^{p}}{p !} \frac{d^{p} w_{\alpha}^{j}\left(x_{j+(-1)^{\alpha}}, u, b_{\alpha}^{(\bar{m}-1)}\right)}{d x^{p}} \\
l_{\alpha}^{(m) j}\left(x_{j}, u\right)= & (-1)^{\alpha} h_{j-1+\alpha} f_{j+(-1)^{\alpha}} \\
& +\sum_{p=2}^{m} \frac{\left[(-1)^{\alpha+1} h_{j-1+\alpha}\right]^{p}}{p !} \frac{d^{p} l_{\alpha}^{j}\left(x_{j+(-1)^{\alpha}}, u, b_{\alpha}^{(\bar{m}-1)}\right)}{d x^{p}} .
\end{aligned}
$$

The following lemma holds.

Lemma 4.1. Let

$$
\begin{gathered}
0<c_{1} \leqslant k(x), \quad k(x) \in Q^{m+1}[0,1], \\
f(x, u) \in \bigcup_{j=1}^{N} C^{m+1}\left(\left[x_{j-1}, x_{j}\right] \times \Omega([0,1], r+\Delta)\right) .
\end{gathered}
$$

Then, the following relations are valid:

$$
\begin{aligned}
V_{\alpha}^{(\bar{m}) j}\left(x_{j}\right)= & (-1)^{\alpha+1} \sum_{p=1}^{\bar{m}} \frac{\left[(-1)^{\alpha+1} h_{j-1+\alpha}\right]^{p}}{p !}\left[\frac{d^{p-1}}{d x^{p-1}} \frac{1}{k(x)}\right]_{x=x_{j+(-1)^{\alpha}}} \\
= & V_{\alpha}^{j}\left(x_{j}\right)+O\left(h_{j-1+\alpha}^{\bar{m}+1}\right), \\
w_{\alpha}^{j}\left(x_{j}, u\right)= & w_{\alpha}^{(\bar{m}) j}\left(x_{j}, u\right) \\
& +(1-\bar{m}+m) \frac{\left[(-1)^{\alpha+1} h_{j-1+\alpha}\right]^{m+1}}{(m+1) !} \frac{d^{m+1} w_{\alpha}^{j}\left(x_{j+(-1)^{\alpha}}, u\right)}{d x^{m+1}}+O\left(h_{j-1+\alpha}^{m+2}\right), \\
l_{\alpha}^{j}\left(x_{j}, u\right)= & l_{\alpha}^{(m) j}\left(x_{j}, u\right)+\frac{\left[(-1)^{\alpha+1} h_{j-1+\alpha}\right]^{m+1}}{(m+1) !} \frac{d^{m+1} l_{\alpha}^{j}\left(x_{j+(-1)^{\alpha}}, u\right)}{d x^{m+1}}+O\left(h_{j-1+\alpha}^{m+2}\right) .
\end{aligned}
$$

Proof. To prove (4.5), we expand the functions on the left-hand side of (4.5) into Taylor series at the points $x_{j+(-1)^{\alpha}}$ representing the residual term in the integral form:

$$
\begin{aligned}
V_{\alpha}^{j}\left(x_{j}\right) & =\sum_{p=1}^{\bar{m}} \frac{\left[(-1)^{\alpha+1} h_{j-1+\alpha}\right]^{p}}{p !} \frac{d^{p} V_{\alpha}^{j}\left(x_{j+(-1)^{\alpha}}\right)}{d x^{p}}+\frac{1}{\bar{m} !} \int_{x_{j+(-1)^{\alpha}}}^{x_{j}}(x-t)^{\bar{m}} \frac{d^{\bar{m}+1} V_{\alpha}^{j}(t)}{d t^{\bar{m}+1}} d t \\
& =V_{\alpha}^{(\bar{m}) j}\left(x_{j}\right)+O\left(h_{j-1+\alpha}^{\bar{m}+1}\right) .
\end{aligned}
$$

Since

$$
\frac{d^{p} V_{\alpha}^{j}\left(x_{j+(-1)^{\alpha}}\right)}{d x^{p}}=(-1)^{\alpha+1}\left[\frac{d^{p-1}}{d x^{p-1}} \frac{1}{k(x)}\right]_{x_{j+(-1)^{\alpha}}}
$$

we arrive at (4.5).

Let us prove by induction the equalities

$$
b_{\alpha}^{(s-1)}=b_{\alpha}+O\left(h_{j-1+\alpha}^{s}\right), \quad s=3,4, \ldots
$$


Let $s=3$. Then we have

$$
\begin{aligned}
b_{\alpha}=w_{\alpha}^{j}\left(x_{j}, u\right) & =-\frac{h_{j-1+\alpha}^{2}}{2} \frac{f_{j+(-1)^{\alpha}}}{k_{j+(-1)^{\alpha}}}+\frac{1}{2 !} \int_{x_{j+(-1)^{\alpha}}}^{x_{j}}\left(x_{j}-t\right)^{2} \frac{d^{3} w_{\alpha}^{j}(t, u)}{d t^{3}} d t \\
& =w_{\alpha}^{(2) j}\left(x_{j}, u\right)+O\left(h_{j-1+\alpha}^{3}\right)=b_{\alpha}^{(2)}+O\left(h_{j-1+\alpha}^{3}\right) .
\end{aligned}
$$

To estimate the residual term, it is necessary that (3.1), (3.2) agree with the estimate of the mixed derivatives of the functions $f\left(x, Y_{\alpha}^{j}\right)$. For this purpose it is necessary that $Y_{\alpha}^{j}(x, u) \in$ $\Omega\left(\left[x_{j-2+\alpha}, x_{j-1+\alpha}\right], r\right)$, and the last is provided with the condition $\left(u_{j}\right)_{j=0}^{N} \in \Omega\left(\hat{\bar{\omega}}_{h}, r\right)$. Assume that relations (4.8) are valid for $s=q-1$ and prove them for $s=q$. Since

$$
\begin{aligned}
b_{\alpha}^{(q)}= & w_{\alpha}^{(q) j}\left(x_{j}, u\right)=-\frac{h_{j-1+\alpha}^{2}}{2} \frac{f_{j+(-1)^{\alpha}}}{k_{j+(-1)^{\alpha}}} \\
& +\sum_{p=3}^{q} \frac{\left[(-1)^{\alpha+1} h_{j-1+\alpha}\right]^{p}}{p !} \frac{d^{p} w_{\alpha}^{j}\left(x_{j+(-1)^{\alpha}}, u, b_{\alpha}^{(q-1)}\right)}{d x^{p}}=-\frac{h_{j-1+\alpha}^{2}}{2} \frac{f_{j+(-1)^{\alpha}}}{k_{j+(-1)^{\alpha}}} \\
& +\sum_{p=3}^{q} \frac{\left[(-1)^{\alpha+1} h_{j-1+\alpha}\right]^{p}}{p !} \frac{d^{p} w_{\alpha}^{j}\left(x_{j+(-1)^{\alpha}}, u, b_{\alpha}+O\left(h_{j-1+\alpha}^{q}\right)\right)}{d x^{p}} \\
= & -\frac{h_{j-1+\alpha}^{2}}{2} \frac{f_{j+(-1)^{\alpha}}}{k_{j+(-1)^{\alpha}}}+\sum_{p=3}^{q} \frac{\left[(-1)^{\alpha+1} h_{j-1+\alpha}\right]^{p}}{p !} \frac{d^{p} w_{\alpha}^{j}\left(x_{j+(-1)^{\alpha}}, u, b_{\alpha}\right)}{d x^{p}}+O\left(h_{j-1+\alpha}^{q+3}\right),
\end{aligned}
$$

we obtain

$$
\begin{aligned}
b_{\alpha}= & -\frac{h_{j-1+\alpha}^{2}}{2} \frac{f_{j+(-1)^{\alpha}}}{k_{j+(-1)^{\alpha}}}+\sum_{p=3}^{q} \frac{\left[(-1)^{\alpha+1} h_{j-1+\alpha}\right]^{p}}{p !} \frac{d^{p} w_{\alpha}^{j}\left(x_{j+(-1)^{\alpha}}, u, b_{\alpha}\right)}{d x^{p}} \\
& +\frac{1}{q !} \int_{x_{j+(-1)^{\alpha}}}^{x_{j}}\left(x_{j}-t\right)^{q} \frac{d^{q+1} w_{\alpha}^{j}\left(t, u, b_{\alpha}\right)}{d t^{q+1}} d t=w_{\alpha}^{(q) j}\left(x_{j}, u\right)+O\left(h_{j-1+\alpha}^{q+1}\right)=b_{\alpha}^{(q)}+O\left(h_{j-1+\alpha}^{q+1}\right) .
\end{aligned}
$$

Moreover

$$
\begin{aligned}
w_{\alpha}^{(m+1) j}\left(x_{j}, u\right)= & -\frac{h_{j-1+\alpha}^{2}}{2} \frac{f_{j+(-1)^{\alpha}}}{k_{j+(-1)^{\alpha}}}+\sum_{p=3}^{m+1} \frac{\left[(-1)^{\alpha+1} h_{j-1+\alpha}\right]^{p}}{p !} \frac{d^{p} w_{\alpha}^{j}\left(x_{j+(-1)^{\alpha}}, u, b_{\alpha}^{(\bar{m}-1)}\right)}{d x^{p}} \\
= & -\frac{h_{j-1+\alpha}^{2}}{2} \frac{f_{j+(-1)^{\alpha}}}{k_{j+(-1)^{\alpha}}}+\sum_{p=3}^{m+1} \frac{\left[(-1)^{\alpha+1} h_{j-1+\alpha}\right]^{p}}{p !} \frac{d^{p} w_{\alpha}^{j}\left(x_{j+(-1)^{\alpha}}, u, b_{\alpha}\right)}{d x^{p}} \\
& +O\left(h_{j-1+\alpha}^{\bar{m}+3}\right), \\
l_{\alpha}^{(m) j}\left(x_{j}, u\right)= & (-1)^{\alpha} h_{j-1+\alpha} f_{j+(-1)^{\alpha}}+\sum_{p=2}^{m} \frac{\left[(-1)^{\alpha+1} h_{j-1+\alpha}\right]^{p}}{p !} \frac{d^{p} l_{\alpha}^{j}\left(x_{j+(-1)^{\alpha}}, u, b_{\alpha}^{\bar{m}-1}\right)}{d x^{p}} \\
= & (-1)^{\alpha} h_{j-1+\alpha} f_{j+(-1)^{\alpha}}+\sum_{p=2}^{m} \frac{\left[(-1)^{\alpha+1} h_{j-1+\alpha}\right]^{p}}{p !} \frac{d^{p} l_{\alpha}^{j}\left(x_{j+(-1)^{\alpha}}, u, b_{\alpha}\right)}{d x^{p}} \\
& +O\left(h_{j-1+\alpha}^{\bar{m}+2}\right) .
\end{aligned}
$$


Therefore

$$
\begin{aligned}
w_{\alpha}^{j}\left(x_{j}, u\right)= & -\frac{h_{j-1+\alpha}^{2}}{2} \frac{f_{j+(-1)^{\alpha}}}{k_{j+(-1)^{\alpha}}}+\sum_{p=3}^{m+1} \frac{\left[(-1)^{\alpha+1} h_{j-1+\alpha}\right]^{p}}{p !} \frac{d^{p} w_{\alpha}^{j}\left(x_{j+(-1)^{\alpha}}, u, b_{\alpha}\right)}{d x^{p}} \\
& +\frac{1}{(m+1) !} \int_{x_{j+(-1)^{\alpha}}}^{x_{j}}\left(x_{j}-t\right)^{m+1} \frac{d^{m+2} w_{\alpha}^{j}\left(t, u, b_{\alpha}\right)}{d t^{m+2}} d t \\
= & w_{\alpha}^{(m+1) j}\left(x_{j}, u\right)+O\left(h_{j-1+\alpha}^{m+2}\right), \\
l_{\alpha}^{j}\left(x_{j}, u\right)= & (-1)^{\alpha} h_{j-1+\alpha} f_{j+(-1)^{\alpha}}+\sum_{p=2}^{m+1} \frac{\left[(-1)^{\alpha+1} h_{j-1+\alpha}\right]^{p}}{p !} \frac{d^{p} l_{\alpha}^{j}\left(x_{j+(-1)^{\alpha}}, u, b_{\alpha}\right)}{d x^{p}} \\
& +\frac{1}{(m+1) !} \int_{x_{j+(-1)^{\alpha}}}^{x_{j}}\left(x_{j}-t\right)^{m+1} \frac{d^{m+2} l_{\alpha}^{j}\left(t, u, b_{\alpha}\right)}{d t^{m+2}} d t \\
= & l_{\alpha}^{(m) j}\left(x_{j}, u\right)+\frac{\left[(-1)^{\alpha+1} h_{j-1+\alpha}\right]^{m+1}}{(m+1) !} \frac{d^{m+1} l_{\alpha}^{j}\left(x_{j+(-1)^{\alpha}}, u, b_{\alpha}\right)}{d t^{m+1}}+O\left(h_{j-1+\alpha}^{m+2}\right) .
\end{aligned}
$$

Let us prove relations (4.6). If $m$ is odd, then $\bar{m}=m+1$, and it follows from (4.8) that

$$
w_{\alpha}^{j}\left(x_{j}, u\right)=w_{\alpha}^{(\bar{m}) j}\left(x_{j}, u\right)+O\left(h_{j-1+\alpha}^{m+2}\right) .
$$

If $m=\bar{m}$ is even, we have

$$
\begin{aligned}
w_{\alpha}^{j}\left(x_{j}, u\right)= & -\frac{h_{j-1+\alpha}^{2}}{2} \frac{f_{j+(-1)^{\alpha}}}{k_{j+(-1)^{\alpha}}}+\sum_{p=3}^{m+1} \frac{\left[(-1)^{\alpha+1} h_{j-1+\alpha}\right]^{p}}{p !} \frac{d^{p} w_{\alpha}^{j}\left(x_{j+(-1)^{\alpha}}, u, b_{\alpha}\right)}{d x^{p}} \\
& +\frac{1}{(m+1) !} \int_{x_{j+(-1)^{\alpha}}}^{x_{j}}\left(x_{j}-t\right)^{m+1} \frac{d^{m+2} w_{\alpha}^{j}\left(t, u, b_{\alpha}\right)}{d t^{m+2}} d t \\
= & w_{\alpha}^{(\bar{m}) j}(x, u)+\frac{\left[(-1)^{\alpha+1} h_{j-1+\alpha}\right]^{m+1}}{(m+1) !} \frac{d^{m+1} w_{\alpha}^{j}\left(x_{j+(-1)^{\alpha}}, u, b_{\alpha}\right)}{d x^{m+1}}+O\left(h_{j-1+\alpha}^{m+2}\right) .
\end{aligned}
$$

The lemma is proved.

Remark 4.1. Instead of the Taylor series expansion method for the numerical solution of the problems (3.1) it is possible to make use of the Runge-Kutta method of an appropriate order of accuracy and to make one step with its help.

Now, instead of the ETDS given by (3.7), (3.8), and (4.1), we can use a truncated TDS of the rank $\bar{m}$ form

$$
\begin{aligned}
\left(a^{(\bar{m})} y_{\bar{x}}^{(\bar{m})}\right)_{\hat{x}} & =-\varphi^{(\bar{m})}\left(x, y^{(\bar{m})}\right), \quad x \in \hat{\omega}_{h}, \quad y^{(\bar{m})}(0)=\mu_{1}, \quad y^{(\bar{m})}(1)=\mu_{2}, \\
a^{(\bar{m})}\left(x_{j}\right) & =\left[\frac{1}{h_{j}} V_{1}^{(\bar{m}) j}\left(x_{j}\right)\right]^{-1}, \\
\varphi^{(\bar{m})}\left(x_{j}, u\right) & =\hbar_{j}^{-1} \sum_{\alpha=1}^{2}(-1)^{\alpha}\left[l_{\alpha}^{(m) j}(x, u)+(-1)^{\alpha} \frac{w_{\alpha}^{(\bar{m}) j}(x, u)}{V_{\alpha}^{(\bar{m}) j}\left(x_{j}\right)}\right] .
\end{aligned}
$$

We need the following lemma to prove the existence and uniqueness of a solution to TDS (4.9) and to establish its accuracy. 
Lemma 4.2. Let the conditions of Lemma 4.1 be satisfied. Then

$$
\begin{aligned}
\left|a^{(\bar{m})}\left(x_{j}\right)-a\left(x_{j}\right)\right| & \leqslant M|h|^{\bar{m}}, \\
\varphi^{(m)}\left(x_{j}, u\right)-\varphi\left(x_{j}, u\right) & =\left\{\left.\frac{h_{j}^{m+1}}{(m+1) !} \frac{d^{m+1} l_{2}^{j-1}(x, u)}{d x^{m+1}}\right|_{x_{j}-0}\right\}_{\hat{x}}+O\left(\frac{h_{j}^{m+2}+h_{j+1}^{m+2}}{\hbar_{j}}\right)
\end{aligned}
$$

if $m$ is odd, and

$$
\begin{aligned}
\varphi^{(m)}\left(x_{j}, u\right) & -\varphi\left(x_{j}, u\right) \\
& =\left\{\left.\frac{h_{j}^{m} k\left(x_{j}-0\right)}{(m+1) !} \frac{d^{m}}{d x^{m}}\left[\frac{l_{2}^{j-1}(x, u)}{k(x)}\right]\right|_{x_{j}-0}\right\}_{\hat{x}}+O\left(\frac{h_{j}^{m+1}+h_{j+1}^{m+1}}{\hbar_{j}}\right)
\end{aligned}
$$

if $m$ is even. Moreover,

$$
\begin{aligned}
&\left|\varphi^{(\bar{m})}\left(x_{j}, u\right)\right| \leqslant K+M|h|, \quad \forall\left(u_{j}\right)_{j=0}^{N} \in \Omega\left(\hat{\bar{\omega}}_{h}, r+\Delta\right), \\
&\left|\varphi^{(\bar{m})}\left(x_{j}, u\right)-\varphi^{(\bar{m})}\left(x_{j}, v\right)\right| \leqslant(L+M|h|)\|u-v\|_{0, \infty, \hat{\omega}_{h}}, \\
& \forall\left(u_{j}\right)_{j=0}^{N},\left(v_{j}\right)_{j=0}^{N} \in \Omega\left(\hat{\bar{\omega}}_{h}, r+\Delta\right),
\end{aligned}
$$

where $M$ is a constant independent of $|h|$.

Proof. Inequality (4.10) follows from (4.5). Prove (4.11) and (4.12). Note that

$$
\begin{aligned}
\varphi^{(\bar{m})}\left(x_{j}, u\right)-\varphi\left(x_{j}, u\right) & =\hbar_{j}^{-1} \sum_{\alpha=1}^{2}(-1)^{\alpha}\left\{l_{\alpha}^{(m) j}\left(x_{j}, u\right)-l_{\alpha}^{j}\left(x_{j}, u\right)\right. \\
& \left.+(-1)^{\alpha}\left[\frac{w_{\alpha}^{(\bar{m}) j}\left(x_{j}, u\right)}{V_{\alpha}^{(\bar{m}) j}\left(x_{j}\right)}-\frac{w_{\alpha}^{j}\left(x_{j}, u\right)}{V_{\alpha}^{j}\left(x_{j}\right)}\right]\right\} .
\end{aligned}
$$

By Lemma 4.1, we have

$$
\begin{aligned}
l_{\alpha}^{j}\left(x_{j}, u\right)- & l_{\alpha}^{(m) j}\left(x_{j}, u\right)=\frac{\left[(-1)^{\alpha+1} h_{j-1+\alpha}\right]^{m+1}}{(m+1) !} \frac{d^{m+1} l_{\alpha}^{j}\left(x_{j+(-1)^{\alpha}}, u\right)}{d x^{m+1}}+O\left(h_{j-1+\alpha}^{m+2}\right), \\
\frac{w_{\alpha}^{j}\left(x_{j}, u\right)}{V_{\alpha}^{j}\left(x_{j}\right)}- & \frac{w_{\alpha}^{(\bar{m}) j}\left(x_{j}, u\right)}{V_{\alpha}^{(\bar{m}) j}\left(x_{j}\right)} \\
= & (1-\bar{m}+m) \frac{\left[(-1)^{\alpha+1} h_{j-1+\alpha}\right]^{m+1}}{(m+1) !} \frac{d^{m+1} w_{\alpha}^{j}\left(x_{j+(-1)^{\alpha}}, u\right)}{d x^{m+1}} \frac{1}{V_{\alpha}^{j}\left(x_{j}\right)} \\
& +O\left(h_{j-1+\alpha}^{m+1}\right) .
\end{aligned}
$$

Combining (4.15), (4.16), (4.17), $V_{1}^{j}\left(x_{j}\right)=\frac{h_{j}}{k_{j-1}}+O\left(h_{j}^{2}\right)$, and $V_{2}^{j}\left(x_{j}\right)=\frac{h_{j+1}}{k_{j+1}}+O\left(h_{j+1}^{2}\right)$, we obtain

$$
\begin{aligned}
\varphi^{(m)}\left(x_{j}, u\right)-\varphi\left(x_{j}, u\right)= & \frac{1}{(m+1) ! \hbar_{j}}\left[h_{j+1}^{m+1} \frac{d^{m+1} l_{2}^{j}\left(x_{j+1}, u\right)}{d x^{m+1}}-h_{j}^{m+1} \frac{d^{m+1} l_{1}^{j}\left(x_{j-1}, u\right)}{d x^{m+1}}\right] \\
& +O\left(\frac{h_{j}^{m+2}+h_{j+1}^{m+2}}{\hbar_{j}}\right)
\end{aligned}
$$


if $m$ is odd, and for $m$ is even, we arrive at the relation

$$
\begin{aligned}
\varphi^{(m)}\left(x_{j}, u\right)-\varphi\left(x_{j}, u\right) & =\frac{1}{(m+1) ! \hbar_{j}}\left[h_{j+1}^{m} k_{j+1} \frac{d^{m+1} w_{2}^{j}\left(x_{j+1}, u\right)}{d x^{m+1}}\right. \\
& \left.-h_{j}^{m} k_{j-1} \frac{d^{m} w_{1}^{j}\left(x_{j-1}, u\right)}{d x^{m+1}}\right]+O\left(\frac{h_{j}^{m+1}+h_{j+1}^{m+1}}{\hbar_{j}}\right) .
\end{aligned}
$$

From (3.3) we have

$$
\begin{aligned}
u(x) & =\hat{u}(x)+w_{1}^{j}(x, u)-\frac{V_{1}^{j}(x)}{V_{1}^{j}\left(x_{j}\right)} w_{1}^{j}\left(x_{j}, u\right) \\
& =\hat{u}(x)+w_{2}^{j-1}(x, u)-\frac{V_{2}^{j-1}(x)}{V_{2}^{j-1}\left(x_{j-1}\right)} w_{2}^{j-1}\left(x_{j}, u\right), \quad x \in\left[x_{j-1}, x_{j}\right] .
\end{aligned}
$$

This reasoning yields the relation

$$
w_{1}^{j}(x, u)=w_{2}^{j-1}(x, u)+\frac{V_{1}^{j}(x)}{V_{1}^{j}\left(x_{j}\right)} w_{1}^{j}\left(x_{j}, u\right)-\frac{V_{2}^{j-1}(x)}{V_{2}^{j-1}\left(x_{j-1}\right)} w_{2}^{j-1}\left(x_{j-1}, u\right), \quad x \in\left[x_{j-1}, x_{j}\right] .
$$

Differentiating the last equality and multiplying it by $k(x)$, we obtain

$$
l_{1}^{j}(x, u)=l_{2}^{j-1}(x, u)+\frac{w_{1}^{j}\left(x_{j}, u\right)+w_{2}^{j-1}\left(x_{j-1}, u\right)}{V_{1}^{j}\left(x_{j}\right)}, \quad x \in\left[x_{j-1}, x_{j}\right]
$$

Since under the conditions of lemma

$$
\begin{aligned}
\frac{d^{m+1} l_{1}^{j}(x, u)}{d x^{m+1}} & =\frac{d^{m+1} l_{2}^{j-1}(x, u)}{d x^{m+1}}, \\
\frac{d^{m+1} w_{1}^{j}(x, u)}{d x^{m+1}} & =\frac{d^{m+1} w_{2}^{j-1}(x, u)}{d x^{m+1}}+\frac{d^{m}}{d x^{m}}\left[\frac{1}{k(x)}\right] \frac{w_{1}^{j}\left(x_{j}, u\right)+w_{2}^{j-1}\left(x_{j-1}, u\right)}{V_{1}^{j}\left(x_{j}\right)} \\
& =\frac{d^{m+1} w_{2}^{j-1}(x, u)}{d x^{m+1}}+O\left(h_{j}\right), \quad x \in\left[x_{j-1}, x_{j}\right]
\end{aligned}
$$

by virtue of $k_{j-1}=k_{j}+O\left(h_{j}\right)$, we have

$$
\begin{gathered}
\frac{d^{m+1} l_{1}^{j}\left(x_{j-1}+0, u\right)}{d x^{m+1}}=\frac{d^{m+1} l_{2}^{j-1}\left(x_{j}-0, u\right)}{d x^{m+1}}+O\left(h_{j}\right), \\
k\left(x_{j-1}+0\right) \frac{d^{m+1} w_{1}^{j}\left(x_{j-1}+0, u\right)}{d x^{m+1}}=k\left(x_{j}-0\right) \frac{d^{m+1} w_{2}^{j-1}\left(x_{j}-0, u\right)}{d x^{m+1}}+O\left(h_{j}\right) .
\end{gathered}
$$

Taking into account (4.20), from (4.18), (4.19) relations (4.11), (4.12) follow. 
Let us prove inequalities (4.13), (4.14). Since

$$
\begin{aligned}
\varphi^{(\bar{m})}\left(x_{j}, u\right)= & \hbar_{j}^{-1} \sum_{\alpha=1}^{2}(-1)^{\alpha}\left[l_{\alpha}^{(m) j}\left(x_{j}, u\right)+(-1)^{\alpha} \frac{w_{\alpha}^{(\bar{m}) j}\left(x_{j}, u\right)}{V_{\alpha}^{(\bar{m}) j}\left(x_{j}\right)}\right] \\
= & \sum_{\alpha=1}^{2}(-1)^{\alpha}\left\{\frac{(-1)^{\alpha}}{2}\left[1+\frac{\left.V_{\alpha}^{(\bar{m}) j}\left(x_{j}\right)-V_{\alpha}^{(1) j}\left(x_{j}\right)\right]}{V_{\alpha}^{(\bar{m}) j}\left(x_{j}\right)}\right] f\left(x_{\left.j+(-1)^{\alpha}, u\right)}\right]\right. \\
& +\frac{1}{\hbar_{j}} \sum_{p=2}^{m} \frac{\left[(-1)^{\alpha+1} h_{j-1+\alpha}\right]^{p}}{p !} \frac{d^{p} l_{\alpha}^{j}\left(x_{j+(-1)^{\alpha}}, u, b_{\alpha}^{(\bar{m}-1)}\right)}{d x^{p}} \\
& \left.+\frac{(-1)^{\alpha}}{\hbar_{j} V_{\alpha}^{(\bar{m}) j}\left(x_{j}\right)} \sum_{p=3}^{\bar{m}} \frac{\left[(-1)^{\alpha+1} h_{j-1+\alpha}\right]^{p}}{p !} \frac{d^{p} w_{\alpha}^{j}\left(x_{j+(-1)^{\alpha}}, u, b_{\alpha}^{(\bar{m}-1)}\right)}{d x^{p}}\right\}
\end{aligned}
$$

we have

$$
\begin{aligned}
& \left|\varphi^{(\bar{m})}\left(x_{j}, u\right)\right| \leqslant K+M|h|, \\
& \left|\varphi^{(\bar{m})}\left(x_{j}, u\right)-\varphi^{(\bar{m})}\left(x_{j}, v\right)\right| \leqslant \sum_{\alpha=1}^{2}\left\{\frac{1}{2}\left|f\left(x_{j+(-1)^{\alpha}}, u\right)-f\left(x_{j+(-1)^{\alpha}}, v\right)\right|\right. \\
& \times\left[1+\frac{\left|V_{\alpha}^{(\bar{m}) j}\left(x_{j}\right)-V_{\alpha}^{j}\left(x_{j}\right)\right|}{\left|V_{\alpha}^{(\bar{m}) j}\left(x_{j}\right)\right|}+\frac{\left|V_{\alpha}^{j}\left(x_{j}\right)-V_{\alpha}^{(1) j}\left(x_{j}\right)\right|}{\left|V_{\alpha}^{(\bar{m}) j}\left(x_{j}\right)\right|}\right]+\frac{1}{\hbar_{j}} \sum_{p=2}^{m} \frac{h_{j-1+\alpha}^{p}}{p !} \\
& \times\left|\frac{d^{p} l_{\alpha}^{j}\left(x_{j+(-1)^{\alpha}}, u, b_{\alpha}^{(\bar{m}-1) j}(u)\right)}{d x^{p}}-\frac{d^{p} l_{\alpha}^{j}\left(x_{j+(-1)^{\alpha}}, v, b_{\alpha}^{(\bar{m}-1) j}(v)\right)}{d x^{p}}\right|+\frac{1}{\hbar_{j}\left|V_{\alpha}^{(\bar{m}) j}\left(x_{j}\right)\right|} \\
& \left.\times \sum_{p=3}^{\bar{m}} \frac{h_{j-1+\alpha}^{p}}{p !}\left|\frac{d^{p} w_{\alpha}^{j}\left(x_{j+(-1)^{\alpha}}, u, b_{\alpha}^{(\bar{m}-1) j}(u)\right)}{d x^{p}}-\frac{d^{p} w_{\alpha}^{j}\left(x_{j+(-1)^{\alpha}}, v, b_{\alpha}^{(\bar{m}-1) j}(u)\right)}{d x^{p}}\right|\right\} .
\end{aligned}
$$

Validity of these inequalities follows from the fact that all mixed derivatives of the function $f(x, u)$ entering into $\frac{d^{p} w_{\alpha}^{j}(x, u)}{d x^{p}}, \frac{d^{p} l_{\alpha}^{j}(x, u)}{d x^{p}}$ will be calculated at the point $\left(x_{j+(-1)^{\alpha}}, u_{j+(-1)^{\alpha}}\right)$, and $\left(u_{j}\right)_{j=0}^{N} \in \Omega\left(\hat{\bar{\omega}}_{h}, r+\Delta\right)$.

Let us prove by induction the inequalities

$$
\left|b_{\alpha}^{(s-1) j}(u)-b_{\alpha}^{(s-1) j}(v)\right| \leqslant B_{s-1}|h|^{2} \cdot\|u-v\|_{0, \infty, \hat{\omega}_{h}}, \quad s=3,4, \ldots,
$$

where $B_{s-1}, s=3,4, \ldots$ are constants independent of $|h|$.

Let $s=3$, then

$$
\begin{aligned}
\left|b_{\alpha}^{(2) j}(u)-b_{\alpha}^{(2) j}(v)\right| & =\left|w_{\alpha}^{(2) j}\left(x_{j}, u\right)-w_{\alpha}^{(2) j}\left(x_{j}, v\right)\right| \leqslant \frac{|h|^{2}}{2 c_{1}} \mid f\left(x_{j+(-1)^{\alpha}}, u\right)-f\left(x_{\left.j+(-1)^{\alpha}, v\right) \mid}\right. \\
& \leqslant \frac{L|h|^{2}}{2 c_{1}}\|u-v\|_{0, \infty, \hat{\omega}_{h}}=B_{2}|h|^{2} \cdot\|u-v\|_{0, \infty, \hat{\omega}_{h}} .
\end{aligned}
$$

Let inequalities (4.21) be valid for $s=q-1$. Using the formula of finite increments, we 
obtain

$$
\begin{aligned}
& \left|b_{\alpha}^{(q) j}(u)-b_{\alpha}^{(q) j}(v)\right|=\left|w_{\alpha}^{(q) j}\left(x_{j}, u\right)-w_{\alpha}^{(q) j}\left(x_{j}, v\right)\right| \\
& \leqslant \frac{h_{j-1+\alpha}^{2}}{2 c_{1}}\left|f\left(x_{j+(-1)^{\alpha}}, u\right)-f\left(x_{j+(-1)^{\alpha}}, v\right)\right| \\
& +\sum_{p=3}^{q} \frac{h_{j-1+\alpha}^{p}}{p !}\left|\frac{d^{p} w_{\alpha}^{j}\left(x_{j+(-1)^{\alpha}}, u, b_{\alpha}^{(q-1) j}(u)\right)}{d x^{p}}-\frac{d^{p} w_{\alpha}^{j}\left(x_{j+(-1)^{\alpha}}, v, b_{\alpha}^{(q-1) j}(v)\right)}{d x^{p}}\right| \\
& \leqslant \frac{L|h|^{2}}{2 c_{1}}\|u-v\|_{0, \infty, \hat{\omega}_{h}}+\sum_{p=3}^{q} \frac{|h|^{p}}{p !}\left\{\left|\frac{\partial}{\partial u}\left[\frac{d^{p} w_{\alpha}^{j}\left(x_{j+(-1)^{\alpha}}, \bar{u}, b_{\alpha}^{(q-1) j}(u)\right)}{d x^{p}}\right]\right|\|u-v\|_{0, \infty, \hat{\omega}_{h}}\right. \\
& \left.+\left|\frac{\partial}{\partial b_{\alpha}^{j}(u)}\left[\frac{d^{p} w_{\alpha}^{j}\left(x_{j+(-1)^{\alpha}}, v, b_{\alpha}^{(q-1) j}(\bar{u})\right)}{d x^{p}}\right]\right|\left|b_{\alpha}^{(q-1) j}(u)-b_{\alpha}^{(q-1) j}(v)\right|\right\} \\
& \leqslant\left(\frac{L|h|^{2}}{2 c_{1}}+M_{q}|h|^{p}\right)\|u-v\|_{0, \infty, \hat{\omega}_{h}}=B_{q}|h|^{2} \cdot\|u-v\|_{0, \infty, \hat{\omega}_{h}}
\end{aligned}
$$

where

$$
\bar{u}=\quad \bar{u}_{j+(-1)^{\alpha}}=\theta u_{j+(-1)^{\alpha}}+(1-\theta) v_{j+(-1)^{\alpha}}, \quad 0<\theta<1 .
$$

The proof of (4.21) is complete.

Then, in view of (4.21) there will be $\bar{u}, \tilde{u}$ such that

$$
\begin{aligned}
& \left|\varphi^{(\bar{m})}\left(x_{j}, u\right)-\varphi^{(\bar{m})}\left(x_{j}, v\right)\right| \leqslant(L+M|h|)\|u-v\|_{0, \infty, \hat{\omega}_{h}} \\
& \quad+\frac{1}{\hbar_{j}\left|V_{\alpha}^{(\bar{m}) j}\left(x_{j}\right)\right|} \sum_{p=3}^{\bar{m}} \frac{h_{j-1+\alpha}^{p}}{p !}\left\{\left|\frac{\partial}{\partial u}\left[\frac{d^{p} w_{\alpha}^{j}\left(x_{j+(-1)^{\alpha}}, \bar{u}, b_{\alpha}^{(\bar{m}-1) j}(u)\right)}{d x^{p}}\right]\right|\|u-v\|_{0, \infty, \hat{\omega}_{h}}\right. \\
& \left.\quad+\left|\frac{\partial}{\partial b_{\alpha}^{j}(u)}\left[\frac{d^{p} w_{\alpha}^{j}\left(x_{j+(-1)^{\alpha}}, v, b_{\alpha}^{(\bar{m}-1) j}(\bar{u})\right)}{d x^{p}}\right]\right|\left|b_{\alpha}^{(\bar{m}-1) j}(u)-b_{\alpha}^{(\bar{m}-1) j}(v)\right|\right\} \\
& \quad+\sum_{p=3}^{m} \frac{h_{j-1+\alpha}^{p}}{p !}\left\{\left|\frac{\partial}{\partial u}\left[\frac{d^{p} l_{\alpha}^{j}\left(x_{j+(-1)^{\alpha}}, \tilde{u}, b_{\alpha}^{(\bar{m}-1) j}(u)\right)}{d x^{p}}\right]\right|\|u-v\|_{0, \infty, \hat{\omega}_{h}}\right. \\
& \left.\quad+\left|\frac{\partial}{\partial b_{\alpha}^{j}(u)}\left[\frac{d^{p} l_{\alpha}^{j}\left(x_{j+(-1)^{\alpha}}, u, b_{\alpha}^{(\bar{m}-1) j}(\tilde{u})\right)}{d x^{p}}\right]\right|\left|b_{\alpha}^{(\bar{m}-1) j}(u)-b_{\alpha}^{(\bar{m}-1) j}(v)\right|\right\} \\
& \quad \leqslant(L+M|h|)\|u-v\|_{0, \infty, \hat{\omega}_{h} .}
\end{aligned}
$$

The proof of the following theorem is based on the preceding statement.

Theorem 4.1. Let the conditions of Theorem 2.1 and Lemma 4.1 be satisfied. Then $\exists h_{0}>0$ such that for $|h| \leqslant h_{0}$ the TDS given by (4.9), (4.2)-(4.4) has a unique solution whose accuracy is characterized by the estimate

$$
\left\|y^{(\bar{m})}-u\right\|_{1, \infty, \hat{\omega}_{h}}^{*}=\max \left\{\left\|y^{(\bar{m})}-u\right\|_{0, \infty, \hat{\omega}_{h}},\left\|k \frac{d y^{(\bar{m})}}{d x}-k \frac{d u}{d x}\right\|_{0, \infty, \hat{\omega}_{h}}\right\} \leqslant M|h|^{\bar{m}},
$$


where

$$
k\left(x_{j}\right) \frac{d y^{(\bar{m})}\left(x_{j}\right)}{d x}=\frac{h_{j} y_{\bar{x}, j}^{(\bar{m})}-w_{1}^{(\bar{m}) j}\left(x_{j}, y^{(\bar{m})}\right)}{V_{1}^{(\bar{m}) j}\left(x_{j}\right)}+l_{1}^{(m) j}\left(x_{j}, y^{(\bar{m})}\right)
$$

and the constant $M$ is dependent on $|h|$.

Proof. Let us show that under the conditions of the theorem TDS of $\bar{m}$ th rank (4.9) has the unique solution $y^{(\bar{m})}(x), x \in \hat{\omega}_{h}$. We use the principle of contraction mapping. Consider the operator equation

$$
\begin{aligned}
y^{(\bar{m})}(x) & =\Re_{h}\left(x,\left(y_{j}^{(\bar{m})}\right)_{j=0}^{N}\right) \\
& =\sum_{\xi \in \hat{\omega}_{h}} \hbar(\xi) G^{(\bar{m})}(x, \xi) \varphi^{(\bar{m})}\left(\xi, y^{(\bar{m})}(\xi)\right)+\frac{V_{2}^{(\bar{m})}(x)}{V_{2}^{(\bar{m})}(0)} \mu_{1}+\frac{V_{1}^{(\bar{m})}(x)}{V_{1}^{(\bar{m})}(1)} \mu_{2},
\end{aligned}
$$

where $G^{(\bar{m})}(x, \xi)$ is the Green function of problem (4.9) of the form (see., for example, [7])

$$
\begin{aligned}
G^{(\bar{m})}(x, \xi) & =\frac{1}{V_{1}^{(\bar{m})}(1)} \begin{cases}V_{1}^{(\bar{m})}(x) V_{2}^{(\bar{m})}(\xi), & 0 \leqslant x \leqslant \xi, \\
V_{2}^{(\bar{m})}(x) V_{1}^{(\bar{m})}(\xi), & \xi \leqslant x \leqslant 1,\end{cases} \\
V_{1}^{(\bar{m})}\left(x_{j}\right) & =\sum_{k=1}^{j} \frac{h_{k}}{a^{(\bar{m})}\left(x_{k}\right)}=\sum_{k=1}^{j} V_{1}^{(\bar{m}) k}\left(x_{k}\right), \\
V_{2}^{(\bar{m})}\left(x_{j}\right) & =\sum_{k=j+1}^{N} \frac{h_{k}}{a^{(\bar{m})}\left(x_{k}\right)}=\sum_{k=j+1}^{N} V_{1}^{(\bar{m}) k}\left(x_{k}\right) .
\end{aligned}
$$

Taking into account the inequalities

$$
G^{(\bar{m})}(x, \xi) \leqslant \frac{1}{c_{1}}+c|h|, \quad\left|G_{\bar{x}}^{(\bar{m})}(x, \xi)\right| \leqslant \frac{1}{c_{1}}+c|h|, \quad c|h|<\frac{1}{c_{1}}
$$

and inequality (4.13), we have

$$
\begin{gathered}
\left\|\Re_{h}\left(x,(u)_{j=0}^{N}\right)-u^{(0)}(x)\right\|_{1, \infty, \hat{\omega}_{h}} \leqslant\left(\frac{1}{c_{1}}+c|h|\right) \sum_{\xi \in \hat{\omega}_{h}} \hbar(\xi)\left|\varphi^{(\bar{m})}(\xi, u)\right| \\
+\left\|\left[\frac{V_{2}^{(\bar{m})}(x)}{V_{1}^{(\bar{m})}(1)}-\frac{V_{2}(x)}{V_{1}(1)}\right] \mu_{1}+\left[\frac{V_{1}^{(\bar{m})}(x)}{V_{1}^{(\bar{m})}(1)}-\frac{V_{1}(x)}{V_{1}(1)}\right] \mu_{2}\right\|_{1, \infty, \hat{\omega}_{h}} .
\end{gathered}
$$


Since

$$
\begin{aligned}
& \left\|\frac{V_{\alpha}^{(\bar{m})}(x)}{V_{1}^{(\bar{m})}(1)}-\frac{V_{\alpha}(x)}{V_{1}(1)}\right\|_{0, \infty, \hat{\omega}_{h}} \leqslant \frac{\left\|V_{\alpha}^{(\bar{m})}(x)-V_{\alpha}(x)\right\|_{0, \infty, \hat{\omega}_{h}}}{\left|V_{1}^{(\bar{m})}(1)\right|}+\frac{\left|V_{1}(1)-V_{1}^{(\bar{m})}(1)\right|\left\|V_{\alpha}(x)\right\|_{0, \infty, \hat{\omega}_{h}}}{\left|V_{1}^{(\bar{m})}(1)\right|\left|V_{1}(1)\right|} \\
& \leqslant \frac{2 \sum_{k=1}^{N}\left|V_{1}^{(\bar{m}) k}\left(x_{k}\right)-V_{1}^{k}\left(x_{k}\right)\right|}{\left|V_{1}^{(\bar{m})}(1)\right|} \leqslant M|h|^{\bar{m}} \\
& \left\|\left[\frac{V_{\alpha}^{(\bar{m})}(x)}{V_{1}^{(\bar{m})}(1)}-\frac{V_{\alpha}(x)}{V_{1}(1)}\right]_{\bar{x}}\right\|_{0, \infty, \hat{\omega}_{h}} \leqslant\left\|\frac{V_{1}^{(\bar{m}) j}(x)}{h_{j} V_{1}^{(\bar{m})}(1)}-\frac{V_{1}^{j}(x)}{h_{j} V_{1}(1)}\right\|_{0, \infty, \hat{\omega}_{h}} \\
& \leqslant\left\|\frac{\left[V_{1}^{(\bar{m}) j}(x)-V_{1}^{j}(x)\right] V_{1}(1)+\left[V_{1}^{(\bar{m})}(1)-V_{1}(1)\right] V_{1}^{j}(x)}{h_{j} V_{1}^{(\bar{m})}(1) V_{1}(1)}\right\|_{0, \infty, \hat{\omega}_{h}} \\
& \leqslant M|h|^{\bar{m}} \text {, }
\end{aligned}
$$

we obtain

$$
\begin{aligned}
\left\|\Re_{h}\left(x,\left(v_{j}\right)_{j=0}^{N}\right)-u^{(0)}(x)\right\|_{1, \infty, \hat{\omega}_{h}} & \leqslant\left(\frac{1}{c_{1}}+c|h|\right)(K+M|h|)+M_{1}|h|^{\bar{m}} \\
& \leqslant r+M_{2}|h| \leqslant r+\Delta, \quad \forall\left(v_{j}\right)_{j=0}^{N} \in \Omega\left(\hat{\bar{\omega}}_{h}, r+\Delta\right),
\end{aligned}
$$

i.e., the operator $\Re_{h}\left(x,\left(v_{j}\right)_{j=0}^{N}\right)$ maps the set $\Omega\left(\hat{\bar{\omega}}_{h}, r+\Delta\right)$ onto itself. Moreover, in view of inequality (4.14)

$$
\begin{aligned}
\left\|\Re_{h}\left(x,\left(u_{j}\right)_{j=0}^{N}\right)-\Re_{h}\left(x,\left(v_{j}\right)_{j=0}^{N}\right)\right\|_{1, \infty, \hat{\omega}_{h}} & \leqslant\left(\frac{1}{c_{1}}+c|h|\right) \max _{\xi \in \hat{\omega}_{h}}\left|\varphi^{(\bar{m})}(\xi, u)-\varphi^{(\bar{m})}(\xi, v)\right| \\
& \leqslant\left(\frac{1}{c_{1}}+c|h|\right)(L+M|h|)\|u-v\|_{1, \infty, \hat{\omega}_{h}} \\
& =q_{2}\|u-v\|_{1, \infty, \hat{\omega}_{h}}, \forall\left(u_{j}\right)_{j=0}^{N},\left(v_{j}\right)_{j=0}^{N} \in \Omega\left(\hat{\bar{\omega}}_{h}, r\right) .
\end{aligned}
$$

If $h_{0}$ is chosen such that $q_{2}=\left(\frac{1}{c_{1}}+c|h|\right)(L+M|h|)<1$, then the mapping $\Re_{h}\left(x,\left(u_{j}\right)_{j=0}^{N}\right)$ is a contraction.

For the error $z(x)=y^{(\bar{m})}(x)-u(x), \quad x \in \hat{\omega}_{h}$ we have the problem

$$
\begin{aligned}
{\left[a^{(\bar{m})}(x) z_{\bar{x}}(x)\right]_{\hat{x}} } & =\varphi(x, u)-\varphi^{(\bar{m})}\left(x, y^{(\bar{m})}\right)+\left[\left(a(x)-a^{(\bar{m})}(x)\right) u_{\bar{x}}(x)\right]_{\hat{x}} \\
z(0) & =z(1)=0 .
\end{aligned}
$$


The solution of this problem can be represented in the form

$$
\begin{aligned}
z(x)= & \sum_{\xi \in \hat{\omega}_{h}} \hbar(\xi) G^{(\bar{m})}(x, \xi)\left\{\varphi(\xi, u)-\varphi^{(\bar{m})}\left(\xi, y^{(\bar{m})}\right)+\left[\left(a^{(\bar{m})}(\xi)-a(\xi)\right) u_{\bar{\xi}}(\xi)\right]_{\hat{\xi}}\right\} \\
= & \sum_{\xi \in \hat{\omega}_{h}^{+}} h(\xi) G_{\bar{\xi}}^{(\bar{m})}(x, \xi)\left[a(\xi)-a^{(\bar{m})}(\xi)\right] u_{\bar{\xi}}(\xi) \\
& +\sum_{\xi \in \hat{\omega}_{h}} \hbar(\xi) G^{(\bar{m})}(x, \xi)\left[\varphi(\xi, u)-\varphi^{(\bar{m})}\left(\xi, y^{(\bar{m})}\right)\right], \\
z_{\bar{x}}(x)= & \sum_{\xi \in \hat{\omega}_{h}^{+}} h(\xi) G_{\bar{x} \bar{\xi}}^{(\bar{m})}(x, \xi)\left[a(\xi)-a^{(\bar{m})}(\xi)\right] u_{\bar{\xi}}(\xi) \\
& +\frac{a(x)-a^{(\bar{m})}(x)}{a^{(\bar{m})}(x)} u_{\bar{x}}(x)+\sum_{\xi \in \hat{\omega}_{h}} \hbar(\xi) G_{\bar{x}}^{(\bar{m})}(x, \xi)\left[\varphi(\xi, u)-\varphi^{(\bar{m})}\left(\xi, y^{(\bar{m})}\right)\right] .
\end{aligned}
$$

If $m$ is odd, then, taking into account (4.11), we obtain from equalities (4.22) and (4.23) that

$$
\begin{aligned}
z(x)= & \sum_{\xi \in \hat{\omega}_{h}^{+}} h(\xi) G_{\bar{\xi}}^{(m+1)}(x, \xi)\left\{\left[a(\xi)-a^{(m+1)}(\xi)\right] u_{\bar{\xi}}(\xi)-\left.\frac{h^{m+1}(\xi)}{(m+1) !} \frac{d^{m+1} l_{2}^{j-1}\left(x, y^{(m)}\right)}{d x^{m+1}}\right|_{x=\xi-0}\right\} \\
& +\sum_{\xi \in \hat{\omega}_{h}} \hbar(\xi) G^{(m+1)}(x, \xi)\left[\varphi^{(m+1)}(\xi, u)-\varphi^{(m+1)}\left(\xi, y^{(m+1)}\right)\right]+O\left(|h|^{m+1}\right), \\
z_{\bar{x}}(x)= & \sum_{\xi \in \hat{\omega}_{h}^{+}} h(\xi) G_{\bar{x} \bar{\xi}}^{(m+1)}(x, \xi)\left\{\left[a(\xi)-a^{(m+1)}(\xi)\right] u_{\bar{\xi}}(\xi)-\left.\frac{h^{m+1}(\xi)}{(m+1) !} \frac{d^{m+1} l_{2}^{j-1}\left(x, y^{(m)}\right)}{d x^{m+1}}\right|_{x=\xi-0}\right\} \\
& +\frac{a(x)-a^{(m+1)}(x)}{a^{(m+1)}(x)} u_{\bar{x}}(x)+\sum_{\xi \in \hat{\omega}_{h}} \hbar(\xi) G_{\bar{x}}^{(m+1)}(x, \xi)\left[\varphi^{(m+1)}(\xi, u)-\varphi^{(m+1)}\left(\xi, y^{(m+1)}\right)\right] \\
& +O\left(|h|^{m+1}\right) .
\end{aligned}
$$

Then,

$$
\begin{aligned}
|z(x)| & \leqslant\left(\frac{1}{c_{1}}+c|h|\right)\left\|a-a^{(m+1)}\right\|_{0,1, \hat{\omega}_{h}^{+}}\left\|u_{\bar{x}}\right\|_{0, \infty, \hat{\omega}_{h}^{+}}+M_{1}|h|^{m+1}+q_{2}\|z\|_{1, \infty, \hat{\omega}_{h}} \\
& \leqslant M|h|^{m+1}+q_{2}\|z\|_{1, \infty, \hat{\omega}_{h}}, \\
\left|z_{\bar{x}}(x)\right| & \leqslant\left(\left|V_{1}^{(m)}(1)\right|^{-1} C+\frac{1}{c_{1}}\right)\left\|a-a^{(m+1)}\right\|_{0,1, \hat{\omega}_{h}^{+}}\left\|u_{\bar{x}}\right\|_{0, \infty, \hat{\omega}_{h}^{+}}+M_{1}|h|^{m+1}+q_{2}\|z\|_{1, \infty, \hat{\omega}_{h}} \\
& \leqslant M|h|^{m+1}+q_{2}\|z\|_{1, \infty, \hat{\omega}_{h}} .
\end{aligned}
$$

If $m$ is even, then, taking into account (4.12), we rewrite equalities (4.22) and (4.23) in the 
form

$$
\begin{aligned}
z(x)= & \sum_{\xi \in \hat{\omega}_{h}^{+}} h(\xi) G_{\bar{\xi}}^{(m)}(x, \xi)\left\{\left[a(\xi)-a^{(m)}(\xi)\right] u_{\bar{\xi}}(\xi)\right. \\
& \left.-\left.h^{m}(\xi) \frac{k(\xi-0)}{(m+1) !} \frac{d^{m}}{d x^{m}}\left[\frac{l_{2}^{j-1}\left(x, y^{(m)}\right)}{k(x)}\right]\right|_{x=\xi-0}\right\} \\
& +\sum_{\xi \in \hat{\omega}_{h}} \hbar(\xi) G^{(m)}(x, \xi)\left[\varphi^{(m)}(\xi, u)-\varphi^{(m)}\left(\xi, y^{(m)}\right)\right]+O\left(|h|^{m}\right), \\
z_{\bar{x}}(x)= & \sum_{\xi \in \hat{\omega}_{h}^{+}} h(\xi) G_{\bar{x} \bar{\xi}}^{(m)}(x, \xi)\left\{\left[a(\xi)-a^{(m)}(\xi)\right] u_{\bar{\xi}}(\xi)-h^{m}(\xi) \frac{k(\xi-0)}{(m+1) !}\right. \\
& \times \frac{d^{m}}{d x^{m}}\left[\frac{l_{2}^{j-1}\left(x, y^{(m)}\right)}{k(x)}\right] \mid \begin{array}{l}
k(x)-a^{(m)}(x) \\
a^{(m)}(x)
\end{array} u_{\bar{x}}(x) \\
& +\sum_{\xi \in \hat{\omega}_{h}} \hbar(\xi) G_{\bar{x}}^{(m)}(x, \xi)\left[\varphi^{(m)}(\xi, u)-\varphi^{(m)}\left(\xi, y^{(m)}\right)\right]+O\left(|h|^{m}\right) .
\end{aligned}
$$

This means that

$$
\begin{aligned}
|z(x)| & \leqslant\left(\frac{1}{c_{1}}+c|h|\right)\left\|a-a^{(m)}\right\|_{0,1, \hat{\omega}_{h}^{+}}\left\|u_{\bar{x}}\right\|_{0, \infty, \hat{\omega}_{h}^{+}}+M|h|^{m}+q_{2}\|z\|_{0, \infty, \hat{\omega}_{h}} \\
& \leqslant M|h|^{m}+q_{2}\|z\|_{1, \infty, \hat{\omega}_{h}}, \\
\left|z_{\bar{x}}(x)\right| & \leqslant\left(\left|\tilde{V}_{1}^{(m)}(1)\right|^{-1} C+\frac{1}{c_{1}}\right)\left\|a-a^{(m)}\right\|_{0,1, \hat{\omega}_{h}^{+}}\left\|u_{\bar{x}}\right\|_{0, \infty, \hat{\omega}_{h}^{+}}+M_{1}|h|^{m}+q_{2}\|z\|_{0, \infty, \hat{\omega}_{h}} \\
& \leqslant M|h|^{m}+q_{2}\|z\|_{1, \infty, \hat{\omega}_{h}} .
\end{aligned}
$$

Hence, we obtain the estimate

$$
\|z\|_{1, \infty, \hat{\omega}_{h}} \leqslant \frac{M}{1-q_{2}}|h|^{\bar{m}} .
$$

By virtue of $q_{2}<1$, the inequality

$$
\|z\|_{1, \infty, \hat{\omega}_{h}} \leqslant M_{1}|h|^{\bar{m}}
$$

follows from this estimate. Since

$$
\begin{aligned}
\left|k(x) \frac{d z}{d x}\right| & \leqslant\left|\frac{1}{V_{1}^{(\bar{m}) j}\left(x_{j}\right)}-\frac{1}{V_{1}^{j}\left(x_{j}\right)}\right| \cdot\left[h_{j}\left|u_{\bar{x}, j}\right|+\left|w_{1}^{j}\left(x_{j}, u\right)\right|\right]+\frac{1}{\left|V_{1}^{(\bar{m}) j}\left(x_{j}\right)\right|} \\
& \times\left[h_{j}\left|y_{\bar{x}, j}^{(\bar{m})}-u_{\bar{x}, j}\right|+\left|w_{1}^{(\bar{m}) j}\left(x_{j}, y^{(\bar{m})}\right)-w_{1}^{j}\left(x_{j}, y^{(\bar{m})}\right)\right|+\left|w_{1}^{j}\left(x_{j}, y^{(\bar{m})}\right)-w_{1}^{j}\left(x_{j}, u\right)\right|\right] \\
& +\left|l_{1}^{(m) j}\left(x, y^{(\bar{m})}\right)-l_{1}^{j}\left(x, y^{(\bar{m})}\right)\right|+\left|l_{1}^{j}\left(x, y^{(\bar{m})}\right)-l_{1}^{j}(x, u)\right| \\
& \leqslant M_{1}|h|^{\bar{m}}+\left[\frac{1}{V_{1}^{(\bar{m}) j}\left(x_{j}\right)}\left|\frac{\partial}{\partial u} w_{1}^{j}(x, u)\right|_{u=\bar{u}}+\left|\frac{\partial}{\partial u} l_{1}^{j}(x, u)\right|_{u=\tilde{u}}\right]\|z\|_{0, \infty, \hat{\omega}_{h}} \\
& \leqslant M|h|^{\bar{m}}, \quad \forall x \in \hat{\omega}_{h},
\end{aligned}
$$

we obtain $\|z\|_{1, \infty,(0,1)}^{*} \leqslant M|h|_{\text {. }}^{\bar{m}}$ The theorem is proved. 
The solution to the nonlinear TDS (4.9), (4.2)-(4.4) of the order of accuracy $\bar{m}$ can be found by the method of successive approximations.

Theorem 4.2. Let the hypotheses of Theorem 4.1 hold. Then the solution of problem (4.9), (4.2)-(4.4) can be found by the method of successive approximations

$$
\begin{aligned}
\left(a^{(\bar{m})} y_{\overline{\bar{m}}}^{(\bar{m}, n)}\right)_{\hat{x}} & =-\varphi^{(\bar{m})}\left(x, y^{(\bar{m}, n-1)}\right), \quad x \in \hat{\omega}_{h}, \\
y^{(\bar{m}, n)}(0) & =\mu_{1}, y^{(\bar{m}, n)}(1)=\mu_{2}, \quad n=1,2, \ldots, \\
y^{(\bar{m}, 0)}(x) & =\frac{V_{2}^{(\bar{m})}(x)}{V_{1}^{(\bar{m})}(1)} \mu_{1}+\frac{V_{1}^{(\bar{m})}(x)}{V_{1}^{(\bar{m})}(1)} \mu_{2}
\end{aligned}
$$

and the estimate

$$
\left\|y^{(\bar{m}, n)}-u\right\|_{1, \infty, \hat{\omega}_{h}}^{*} \leqslant M\left(|h|^{\bar{m}}+q_{2}^{n}\right)
$$

is valid, where

$$
k\left(x_{j}\right) \frac{d y^{(\bar{m}, n)}\left(x_{j}\right)}{d x}=\frac{h_{j} y_{\bar{x}, j}^{(\bar{m}, n)}-w_{1}^{(\bar{m}) j}\left(x_{j,} y^{(\bar{m}, n)}\right)}{V_{1}^{(\bar{m}) j}\left(x_{j}\right)}+l_{1}^{(m) j}\left(x_{j}, y^{(\bar{m}, n)}\right),
$$

the constant $M$ is independent of $|h|, m, n$ and $q_{2}=q+M|h|<1$.

Proof. By Theorem 4.1, we have

$$
\begin{aligned}
\left\|y^{(\bar{m}, n)}-u\right\|_{1, \infty, \hat{\omega}_{h}}^{*} & \leqslant\left\|y^{(\bar{m})}-u\right\|_{1, \infty, \hat{\omega}_{h}}^{*}+\left\|y^{(\bar{m}, n)}-y^{(\bar{m})}\right\|_{1, \infty, \hat{\omega}_{h}}^{*} \\
& \leqslant M|h|^{\bar{m}}+\left\|y^{(\bar{m}, n)}-y^{(\bar{m})}\right\|_{1, \infty, \hat{\omega}_{h}}^{*} .
\end{aligned}
$$

Thus, the sequence of approximations

$$
y^{(\bar{m}, n)}(x)=\Re_{h}\left(x,\left(y^{(\bar{m}, n-1)}\right)_{j=0}^{N}\right), \quad x \in \hat{\omega}_{h}, \quad n=1,2, \ldots
$$

converges (see the proof of Theorem 3), and we have the following estimate of the convergence rate:

$$
\left\|y^{(\bar{m}, n)}-y^{(\bar{m})}\right\|_{1, \infty, \hat{\omega}_{h}} \leqslant \frac{q_{2}^{n}}{1-q_{2}}(r+\Delta) .
$$

Then, taking into account estimate (4.14), we have

$$
\begin{aligned}
& \left|k(x) \frac{d y^{(\bar{m}, n)}(x)}{d x}-k(x) \frac{d y^{(\bar{m})}(x)}{d x}\right| \\
\leqslant & \frac{1}{\left|V_{1}^{(\bar{m}) j}\left(x_{j}\right)\right|}\left[h_{j}\left|y_{\bar{x}, j}^{(\bar{m}, n)}-y_{\bar{x}, j}^{(\bar{m})}\right|+\left|w_{1}^{(\bar{m}) j}\left(x_{j}, y^{(\bar{m}, n)}\right)-w_{1}^{(\bar{m}) j}\left(x_{j}, y^{(\bar{m})}\right)\right|\right] \\
& +\left|l_{1}^{(m) j}\left(x, y^{(\bar{m}, n)}\right)-l_{1}^{(m) j}\left(x, y^{(\bar{m})}\right)\right| \\
\leqslant & M_{1}|| y_{\bar{x}}^{(\bar{m}, n)}-\left.y_{\bar{x}}^{(\bar{m})}\right|_{0, \infty, \hat{\omega}_{h}^{+}}+\left[\frac{1}{\left|V_{1}^{(\bar{m}) j}\left(x_{j}\right)\right|}\left|\frac{\partial}{\partial u} w_{1}^{(\bar{m}) j}(x, u)\right|_{u=\bar{y}^{(m)}}\right]\left\|y^{(\bar{m}, n)}-y^{(\bar{m})}\right\|_{0, \infty, \hat{\omega}_{h}} \leqslant M\left\|y^{(\bar{m}, n)}-y^{(\bar{m})}\right\|_{1, \infty, \hat{\omega}_{h}} .
\end{aligned}
$$

This means that

$$
\left\|y^{(\bar{m}, n)}-y^{(\bar{m})}\right\|_{1, \infty, \hat{\omega}_{h}}^{*} \leqslant M q_{2}^{n} .
$$

Inequalities (4.25), (4.26) imply estimate (4.24). 


\section{Numerical example}

Consider the boundary-value problem from [5]

$$
\frac{d^{2} u}{d x^{2}}=e^{u}, \quad u(0)=0, \quad u(1)=-\ln \cos ^{2}\left(\frac{1}{\sqrt{2}}\right)
$$

with the exact solution $u(x)=-\ln \cos ^{2}(x / \sqrt{2})$. To evaluate the convergence rate in practice, the following quantities are introduced:

$$
e r=\left\|z^{(m)}\right\|_{1, \infty, \omega_{h}}^{*}=\left\|y^{(m)}-u\right\|_{1, \infty, \omega_{h}}^{*}, \quad p=\log _{2} \frac{\left\|z^{(m)}\right\|_{1, \infty, \omega_{h}}^{*}}{\left\|z^{(m)}\right\|_{1, \infty, \omega_{h / 2}}^{*}} .
$$

The results of the calculations by the methods of the fourth-order $(m=3)$ and six-order $(m=5)$ of the accuracy are presented in the Tables 1,2 respectively. It is seen that the numerical experiment confirms the results of the theoretical analysis.

Table 1.

\begin{tabular}{|l|l|l|}
\hline$N$ & er & $p$ \\
\hline 8 & $0,5431(-4)$ & \\
\hline 16 & $0,3449(-5)$ & 4,0 \\
\hline 32 & $0,2179(-6)$ & 4,0 \\
\hline 64 & $0,1371(-7)$ & 4,0 \\
\hline 128 & $0,8596(-9)$ & 4,0 \\
\hline 256 & $0,5380(-10)$ & 4,0 \\
\hline 512 & $0,3360(-11)$ & 4,0 \\
\hline
\end{tabular}

Table 2.

\begin{tabular}{|l|l|l|}
\hline$N$ & $e r$ & $p$ \\
\hline 8 & $0,4777(-6)$ & \\
\hline 16 & $0,7413(-8)$ & 6,0 \\
\hline 32 & $0,1161(-9)$ & 6,0 \\
\hline 64 & $0,1817(-11)$ & 6,0 \\
\hline
\end{tabular}

\section{References}

[1] A. Kufner and S. Fucik, Nonlinear Differential Equations, Elsevier Scientific Publishing Company, Amsterdam-Oxford-New York, 1980.

[2] M. V. Kutniv, Accurate three-point difference schemes for second-order monotone ordinary differential equations and their implementation, Comput. Math. Math. Phys., 40 (2000), No. 3, pp. 368-382.

[3] M. V. Kutniv, Three-point difference schemes of high accuracy order for systems of nonlinear ordinary differential equations of the second order, Comput. Math. Math. Phys., 41 (2001), No. 6, pp. 860-873. 
[4] M. V. Kutniv, High-order accurate three-point difference schemes for systems of second-order ordinary differential equations with a monotone operator, Comput. Math. Math. Phys., 42 (2002), No. 5, pp. 724-738.

[5] M. V. Kutniv, V. L. Makarov, and A. A. Samarskii, Accurate three-point difference schemes for secondorder nonlinear ordinary differential equations and their implementation, Comput. Math. Math. Phys., 39 (1999), No. 1, pp. 40-45.

[6] V. L. Makarov and A. A. Samarskii, Exact three-point difference schemes for nonlinear ordinary differential equations of second order, and their realization, Soviet Math. Dokl., 41 (1990), No. 3, pp. 495-500.

[7] A. A. Samarskii, Introduction into the Theory of Difference Schemes, Nauka, Moscow, 1971.

[8] A. A. Samarskii, R. D. Lazarov, and V. L. Makarov, Difference Schemes for Differential Equations with Generalized Solutions, Nauka, Moscow, 1987.

[9] V. A. Trenogin, Functional Analysis, Nauka, Moscow, 1980.

Received 18 Dec. 2002

Revised 17 Jan. 2003 